ESAIM: M2AN 48 (2014) 325-346

DOI: $10.1051 / \mathrm{m} 2 \mathrm{an} / 2013110$
ESAIM: Mathematical Modelling and Numerical Analysis

www.esaim-m2an.org

\title{
AN OPTIMAL QUANTITATIVE TWO-SCALE EXPANSION IN STOCHASTIC HOMOGENIZATION OF DISCRETE ELLIPTIC EQUATIONS
}

\author{
Antoine Gloria ${ }^{1}$, Stefan Neukamm ${ }^{2,3}$ and Felix Otto ${ }^{2}$
}

\begin{abstract}
We establish an optimal, linear rate of convergence for the stochastic homogenization of discrete linear elliptic equations. We consider the model problem of independent and identically distributed coefficients on a discretized unit torus. We show that the difference between the solution to the random problem on the discretized torus and the first two terms of the two-scale asymptotic expansion has the same scaling as in the periodic case. In particular the $L^{2}$-norm in probability of the $H^{1}$-norm in space of this error scales like $\varepsilon$, where $\varepsilon$ is the discretization parameter of the unit torus. The proof makes extensive use of previous results by the authors, and of recent annealed estimates on the Green's function by Marahrens and the third author.
\end{abstract}

Mathematics Subject Classification. 35B27, 39A70, 60H25, 60F99.

Received August 3, 2013.

Published online January 21, 2014.

\section{INTRODUCTION}

We establish a linear rate of convergence for the stochastic homogenization of discrete linear elliptic equations, which is optimal. Before we turn to the stochastic case, let us recall some standard analogous results in the periodic case. Let $A$ be a uniformly elliptic and bounded symmetric matrix field on the unit torus $\mathbb{T}:=(\mathbb{R} / \mathbb{Z})^{d}$, $D \subset \mathbb{R}^{d}$ be a smooth domain, and $f$ be a smooth function. Let $\varepsilon>0$. It is well-known that the unique weak solution $u_{\varepsilon} \in H_{0}^{1}(D)$ of the linear elliptic equation

$$
\left\{\begin{aligned}
-\nabla \cdot A(\dot{\bar{\varepsilon}}) \nabla u_{\varepsilon} & =f \text { in } D, \\
u_{\varepsilon} & =0 \text { on } \partial D
\end{aligned}\right.
$$

converges weakly in $H^{1}(D)$ as $\varepsilon \rightarrow 0$ to the unique weak solution $u_{\text {hom }} \in H_{0}^{1}(D)$ of the homogenized equation

$$
\left\{\begin{aligned}
-\nabla \cdot A_{\text {hom }} \nabla u_{\text {hom }} & =f \text { in } D \\
u_{\text {hom }} & =0 \text { on } \partial D .
\end{aligned}\right.
$$

Keywords and phrases. Stochastic homogenization, homogenization error, quantitative estimate.

1 Université Libre de Bruxelles (ULB) Brussels, Belgium and Project-team SIMPAF Inria Lille - Nord Europe Villeneuve d'Ascq, France. agloria@ulb.ac.be

2 Max-Planck-Institut für Mathematik in den Naturwissenschaften Leipzig, Germany. neukamm@mis.mpg.de; otto@mis.mpg.de

3 Present address: Weierstraß-Institut Berlin, Germany. 
The homogenized matrix $A_{\text {hom }}$ is symmetric and characterized for all $\xi \in \mathbb{R}^{d}$ by

$$
\xi \cdot A_{\mathrm{hom}} \xi=\int_{\mathbb{T}}\left(\nabla \phi_{\xi}+\xi\right) \cdot A\left(\nabla \phi_{\xi}+\xi\right)
$$

where $\phi_{\xi}$ is the unique weak solution in $H^{1}(\mathbb{T})$ with vanishing mean of the periodic corrector equation in direction $\xi$ :

$$
-\nabla \cdot A\left(\nabla \phi_{\xi}+\xi\right)=0 \text { in } \mathbb{T} .
$$

From a two-scale expansion, one formally expects for all $x$ in the interior of $D$ :

$$
u_{\varepsilon}(x)=u_{\mathrm{hom}}(x)+\varepsilon \sum_{j=1}^{d} \phi_{j}\left(\frac{x}{\varepsilon}\right) \nabla_{j} u_{\mathrm{hom}}(x)+o(\varepsilon),
$$

where the $\phi_{j}$ are the correctors in the canonical directions $\mathbf{e}_{j}$ (extended by periodicity to $\mathbb{R}^{d}$ ). This identity cannot hold at the boundary since the correctors $\phi_{j}$ do not satisfy the homogeneous Dirichlet boundary conditions: there is indeed a boundary layer. Yet, for all $\tilde{D}$ compactly supported in $D$, Allaire and Amar [1], Theorem 2.3 proved the following rigorous version of (1.3) if the coefficients $A$ are Hölder continuous:

$$
\left\|u_{\varepsilon}-u_{\mathrm{hom}}-\varepsilon \sum_{j=1}^{d} \phi_{j}(\dot{\bar{\varepsilon}}) \nabla_{j} u_{\mathrm{hom}}\right\|_{H^{1}(\tilde{D})} \leq C \varepsilon,
$$

where the multiplicative constant $C$ only depends on $\tilde{D}, D$, the Hölder exponent and norm of $A$, and on the $C^{3}$-norm of $u_{\text {hom }}$ (which is smooth since $f$ and $D$ are smooth). This result relies on a previous work by Avellaneda and Lin [2], Theorem 5, who proved under similar assumptions that

$$
\left\|u_{\varepsilon}-u_{\mathrm{hom}}\right\|_{L^{\infty}(D)} \leq C \varepsilon
$$

These are bounds on the homogenization error.

In the stochastic case, we consider a matrix field $A$ that is stationary and ergodic, in place of periodic. We refer the reader to [18] for details. In order to obtain quantitative estimates in the spirit of (1.4) and (1.5), one has to make assumptions on the statistics of $A$ in addition to ergodicity. There are few results in the literature on quantitative estimates of the homogenization error for elliptic equations in divergence form in the stochastic case. In [19], Theorem 3.1, Yurinskiu proved for algebraically decaying correlations that for all $d>2$, there exists some Hölder exponent $\gamma>0$ and a function $T$ of $\varepsilon$ such that

$$
\left\langle\left\|u_{\varepsilon}-u_{\mathrm{hom}}-\varepsilon \sum_{j=1}^{d} \phi_{T(\varepsilon), j}(\dot{\bar{\varepsilon}}) \nabla_{j} u_{\mathrm{hom}}\right\|_{H^{1}(D)}^{2}\right\rangle^{1 / 2} \leq C \varepsilon^{\gamma},
$$

where $\phi_{T, j}$ is the modified corrector, which is the stationary, almost sure solution to

$$
T^{-1} \phi_{T, j}-\nabla \cdot A\left(\nabla \phi_{T, j}+\mathbf{e}_{j}\right)=0 \text { in } \mathbb{R}^{d} .
$$

This equation is an approximation of the corrector equation when $T \rightarrow \infty$. This is the first quantitative result in stochastic homogenization. Note that a formal linearization in the case of small ellipticity contrast $\lambda \uparrow 1$ yields $\gamma=1$ for $d>2$. Besides not covering dimension $d=2$, the work by Yurinskiu does not allow to reach the scaling $\gamma=1$, even in the case of small ellipticity constrast (and for domains $\tilde{D}$ compactly included in $D$ ). 
In this article, we simplify the above setting with three respects:

(a) We treat finite difference equations instead of continuum partial differential equations. More precisely, we replace the differential operator in (1.1) by the finite difference operator $\nabla_{\varepsilon}^{*} \boldsymbol{a}(\dot{\bar{\varepsilon}}) \nabla_{\varepsilon}$ which acts on discrete functions defined on the scaled lattice $\varepsilon \mathbb{Z}^{d}, \varepsilon>0$. Here $\nabla_{\varepsilon}$ and $\nabla_{\varepsilon}^{*}$ are finite difference approximations of the continuum gradient $\nabla$ and continuum divergence operator $-\nabla \cdot$, and are defined for scalar fields $v: \varepsilon \mathbb{Z}^{d} \rightarrow \mathbb{R}$ and vector fields, $g=\left(g_{1}, \ldots, g_{d}\right): \varepsilon \mathbb{Z}^{d} \rightarrow \mathbb{R}^{d}$ as follows:

$$
\begin{aligned}
& \nabla_{\varepsilon, i} v(y)=\varepsilon^{-1}\left(v\left(y+\varepsilon \mathbf{e}_{i}\right)-v(y)\right), \quad \nabla_{\varepsilon, i}^{*} v(y)=\varepsilon^{-1}\left(v\left(y-\varepsilon \mathbf{e}_{i}\right)-v(y)\right), \\
& \nabla_{\varepsilon} v=\left(\nabla_{\varepsilon, 1} v, \ldots, \nabla_{\varepsilon, d} v\right), \quad \nabla_{\varepsilon}^{*} g=\sum_{i=1}^{d} \nabla_{\varepsilon, i}^{*} g_{i} .
\end{aligned}
$$

The coefficients of the operator $\nabla_{\varepsilon}^{*} \boldsymbol{a}(\dot{\bar{\varepsilon}}) \nabla_{\varepsilon}$ are given by a randomly chosen matrix field $\boldsymbol{a}: \mathbb{Z}^{d} \rightarrow \mathbb{R}^{d \times d}$. We assume that $\boldsymbol{a}$ is diagonal and uniformly elliptic in the sense that

$$
\boldsymbol{a}(x) \in \Omega_{0}:=\left\{\left(\begin{array}{ccc}
\boldsymbol{a}_{1} & & 0 \\
& \ddots & \\
& \ddots & \boldsymbol{a}_{d}
\end{array}\right) \mid \lambda \leq \boldsymbol{a}_{j} \leq 1, j=1, \ldots, d\right\}
$$

for all $x \in \mathbb{Z}^{d}$. Above, $\lambda>0$ is a (deterministic) ellipticity constant and fixed throughout the paper.

(b) In order to avoid boundary layers, we consider a problem on the (discretized) unit torus $\mathbb{T}_{\varepsilon}:=(\varepsilon \mathbb{Z} / \mathbb{Z})^{d}$ and assume w.l.o.g. that the grid size $\varepsilon$ is the inverse of a large integer $L:=\varepsilon^{-1} \in \mathbb{N}$. The equation under consideration is the following finite difference equation with random coefficients

$$
\nabla_{\varepsilon}^{*} \boldsymbol{a}(\dot{\bar{\varepsilon}}) \nabla_{\varepsilon} u_{\varepsilon}=f_{\varepsilon} \text { on } \mathbb{T}_{\varepsilon}, \quad \sum_{\mathbb{T}_{\varepsilon}} u_{\varepsilon}=0
$$

where $f_{\varepsilon}: \mathbb{T}_{\varepsilon} \rightarrow \mathbb{R}$ is a deterministic r.h.s. with zero mean, and should be viewed as a discretization of a continuum r.h.s. $f \in L^{2}(\mathbb{T})$. Note that on $\mathbb{T}_{\varepsilon}$ we have by periodicity the discrete integration by parts formula

$$
\sum_{\mathbb{T}_{\varepsilon}} \nabla_{\varepsilon} v \cdot g=\sum_{\mathbb{T}_{\varepsilon}} v \nabla_{\varepsilon}^{*} g \quad \text { for all } v: \mathbb{T}_{\varepsilon} \rightarrow \mathbb{R} \text { and } g: \mathbb{T}_{\varepsilon} \rightarrow \mathbb{R}^{d} .
$$

(c) We consider the simplest possible statistics, namely independent and identically distributed (i.i.d.) coefficients. Since (1.9) is posed on the discretized unit torus $\mathbb{T}_{\varepsilon}, \varepsilon^{-1}=L \in \mathbb{N}$, we in fact consider the periodic i.i.d. ensemble which is constructed as follows: for a fixed measure $\beta$ on our matrix space $\Omega_{0}$ ("single-site measure") and for fixed size $L$ of the discrete, rescaled torus $\mathbb{T}_{L}:=(\mathbb{Z} / L \mathbb{Z})^{d}=\frac{1}{\varepsilon} \mathbb{T}_{\varepsilon}$, we shall throughout the paper denote by $\langle\cdot\rangle$ the periodic i.i.d. ensemble on

$$
\Omega_{L}=\left\{\boldsymbol{a}: \mathbb{Z}^{d} \rightarrow \Omega_{0} \mid \forall z \in \mathbb{Z}^{d} \boldsymbol{a}(\cdot+L z)=\boldsymbol{a}\right\} \widehat{=} \Omega_{0}^{\mathbb{T}_{L}} .
$$

For a random variable, i.e. a measurable function $\zeta: \Omega_{L} \rightarrow \mathbb{R}$, it is given by the product measure

$$
\langle\zeta\rangle=\prod_{x \in \mathbb{T}_{L}} \int_{\Omega_{0}} \zeta(\boldsymbol{a}) \beta(d \boldsymbol{a}(x))
$$

Evidently, our i.i.d. ensemble is stationary in the sense that for all shifts $y \in \mathbb{Z}^{d}$ the random (periodic) tensor fields $\boldsymbol{a}$ and $\boldsymbol{a}(\cdot+y)$ have the same distribution in $\Omega_{L}$.

In the above setting the qualitative theory of stochastic homogenization applies (see e.g. Kozlov [12], Papanicolaou and Varadhan [18] and Künnemann [13], Theorem 4): there exists a deterministic, symmetric, positive definite $d \times d$ matrix $\boldsymbol{a}_{\text {hom }}$ (only depending on the single-site probability measure $\beta$ ) such that the following statement is true: suppose that $f_{\varepsilon}$ converges (in a discrete $H^{-1}$-norm) to some function $f \in L^{2}(\mathbb{T}$ ) 
with $\int_{\mathbb{T}} f=0$. Let $u_{\varepsilon}$ be the unique (random) solution with vanishing mean to (1.9), and let $u_{\text {hom }} \in H^{1}(\mathbb{T})$ be the unique solution to the continuum homogenized equation

$$
-\nabla \cdot \boldsymbol{a}_{\mathrm{hom}} \nabla u_{\mathrm{hom}}=f \text { in } \mathbb{T}, \quad \int_{\mathbb{T}} u_{\mathrm{hom}}=0 .
$$

Then $u_{\varepsilon}$ converges almost surely (weakly in the discrete $H^{1}$-norm) to $u_{\text {hom }}$.

We are interested in estimates on the speed of convergence of $u_{\varepsilon}$ to $u_{\text {hom }}$. The error $u_{\varepsilon}-u_{\text {hom }}$ consists of two contributions that are of different nature, namely the homogenization error and the discretization error. The latter is purely deterministic and introduced by approximating the deterministic, continuum, homogenized equation (1.11) by the deterministic, homogenized, finite difference equation

$$
\nabla_{\varepsilon}^{*} \boldsymbol{a}_{\mathrm{hom}} \nabla_{\varepsilon} u_{0, \varepsilon}=f_{\varepsilon} \text { in } \mathbb{T}_{\varepsilon}, \quad \sum_{\mathbb{T}_{\varepsilon}} u_{0, \varepsilon}=0
$$

where $f_{\varepsilon} \in L^{2}\left(\mathbb{T}_{\varepsilon}\right)$ denotes a suitable approximation of the continuum r.h.s. $f$. The discretization error is well-studied and well-understood (see for instance [11]).

In this paper we focus on the homogenization error that monitors the difference between the random, variablecoefficient equation (1.9) and the deterministic, constant-coefficient equation (1.12). The main result of this paper is the upcoming quantitative two-scale expansion estimate. We quantify the error in the discrete $L^{2}\left(\mathbb{T}_{\varepsilon}\right)$ and $H^{1}\left(\mathbb{T}_{\varepsilon}\right)$-norms, which are defined for $v: \mathbb{T}_{\varepsilon} \rightarrow \mathbb{R}$ as

$$
\|v\|_{L^{2}\left(\mathbb{T}_{\varepsilon}\right)}=\sqrt{\varepsilon^{d} \sum_{\mathbb{T}_{\varepsilon}} v^{2}}, \quad\|v\|_{H^{1}\left(\mathbb{T}_{\varepsilon}\right)}=\sqrt{\varepsilon^{d} \sum_{\mathbb{T}_{\varepsilon}}\left(v^{2}+\left|\nabla_{\varepsilon} v\right|^{2}\right)} .
$$

Theorem 1.1. Let $d \geq 2$ and $\boldsymbol{a}$ be i.i.d. coefficients. Then there exists a deterministic, symmetric, positive definite matrix $\boldsymbol{a}_{\mathrm{hom}} \in \mathbb{R}^{d \times d}$ (only depending on $\beta$ and $d$ ) with the following property.

Given $\varepsilon>0$ with $\varepsilon^{-1}=L \in \mathbb{N}$, and a r.h.s.

$$
f_{\varepsilon}: \mathbb{T}_{\varepsilon} \rightarrow \mathbb{R} \quad \text { with } \sum_{\mathbb{T}_{\varepsilon}} f_{\varepsilon}=0
$$

let $u_{\varepsilon}: \Omega_{L} \times \mathbb{T}_{\varepsilon} \rightarrow \mathbb{R}$ and $u_{0, \varepsilon}: \mathbb{T}_{\varepsilon} \rightarrow \mathbb{R}$ be the unique solutions to (1.9) and (1.12), respectively. Then

$$
\left\langle\left\|u_{\varepsilon}-u_{0, \varepsilon}-\varepsilon \sum_{j=1}^{d} \phi_{j}(\dot{\bar{\varepsilon}}) \nabla_{\varepsilon, j} u_{0, \varepsilon}\right\|_{H^{1}\left(\mathbb{T}_{\varepsilon}\right)}^{2}\right\rangle^{1 / 2} \lesssim \varepsilon\left\|f_{\varepsilon}\right\|_{L^{2}\left(\mathbb{T}_{\varepsilon}\right)}\left\{\begin{array}{ll}
\left(\ln \frac{1}{\varepsilon}\right)^{1 / 2} & \text { for } d=2 \\
1 & \text { for } d>2
\end{array},\right.
$$

where $\phi_{1}, \ldots, \phi_{d}$ are the periodic correctors associated with the periodic i.i.d. ensemble $\langle\cdot\rangle$ via the periodic corrector equation (see (2.3) below). The multiplicative constant in (1.13) only depends on the constant of ellipticity $\lambda$, and the dimension $d$.

As a corollary we get:

Corollary 1.2. In the situation of Theorem 1.1 we have

$$
\left\langle\left\|u_{\varepsilon}-u_{0, \varepsilon}\right\|_{L^{2}\left(\mathbb{T}_{\varepsilon}\right)}^{2}\right\rangle^{1 / 2} \lesssim \varepsilon\left\|f_{\varepsilon}\right\|_{L^{2}\left(\mathbb{T}_{\varepsilon}\right)}\left\{\begin{array}{ll}
\left(\ln \frac{1}{\varepsilon}\right)^{1 / 2} & \text { for } d=2 \\
1 & \text { for } d>2
\end{array},\right.
$$

The multiplicative constant in this estimate only depends on $\lambda$ and $d$. 
Note that a bound similar to (1.14) (with however a suboptimal exponent) was recentlty obtained by Conlon and Spencer in [4], in the case of the whole space and a massive term. This result complements the estimates of the quantity $u_{\varepsilon}-\left\langle u_{\varepsilon}\right\rangle$ obtained in [5], Theorem 2, and in their optimal form in [14], Corollaries 2 and 3. In particular, the annealed estimates of [14], Theorem 1, see Lemma 3.3 further, on the gradient of the (periodic) Green's function, combined with the argument leading to [5], Theorem 2, allow one to prove that

$$
\left\langle\left\|u_{\varepsilon}-\left\langle u_{\varepsilon}\right\rangle\right\|_{L^{2}\left(\mathbb{T}_{\varepsilon}\right)}^{2}\right\rangle^{1 / 2} \lesssim \varepsilon\left\|f_{\varepsilon}\right\|_{L^{2}\left(\mathbb{T}_{\varepsilon}\right)}\left\{\begin{array}{ll}
\left(\ln \frac{1}{\varepsilon}\right)^{1 / 2} & \text { for } d=2 \\
1 & \text { for } d>2
\end{array} .\right.
$$

The combination of this estimate with Corollary 1.2 then yields an estimate of the difference between the solution $u_{0, \varepsilon}$ of the problem with constant coefficients and the expectation $\left\langle u_{\varepsilon}\right\rangle$ of the solution of the original problem - i.e. the systematic error:

$$
\left\|u_{0, \varepsilon}-\left\langle u_{\varepsilon}\right\rangle\right\|_{L^{2}\left(\mathbb{T}_{\varepsilon}\right)} \lesssim \varepsilon\left\|f_{\varepsilon}\right\|_{L^{2}\left(\mathbb{T}_{\varepsilon}\right)}\left\{\begin{array}{ll}
\left(\ln \frac{1}{\varepsilon}\right)^{1 / 2} & \text { for } d=2 \\
1 & \text { for } d>2
\end{array} .\right.
$$

It is rather surprising that we have to go through the $H^{1}$-norm in order to control this systematic error, or conversely that we do not have to estimate this term in order to prove the (seemingly stronger) statement of Theorem 1.1. Compared to the work [19] by Yurinskiü, Theorem 1.1 covers dimension $d=2$ and gives optimal estimates (in terms of scaling in $\varepsilon$ ) in any dimension, as can be seen by considering the regime of small ellipticity contrast. Note that for $d=1$, the scaling is different: (1.14) is expected to be replaced by

$$
\left\langle\left\|u_{\varepsilon}-u_{0, \varepsilon}\right\|_{L^{2}\left(\mathbb{T}_{\varepsilon}\right)}^{2}\right\rangle^{1 / 2} \lesssim \sqrt{\varepsilon}\left\|f_{\varepsilon}\right\|_{L^{2}\left(\mathbb{T}_{\varepsilon}\right)}
$$

as explicity checked in the continuum setting by Bourgeat and Piatnitski in [3]. As opposed to these works, the present analysis heavily relies on the use of a spectral gap estimate in the probability space. We refer the reader to [6] for relevant references on the subject.

Remark 1.3. The reason why we consider the discrete setting is the following: in the proof of Theorem 1.1 we make extensive use of recent, quantitative results that we obtained in a series of paper $[6,9,10,14]$ in the discrete setting. The extension of some results to the continuum setting is currently under investigation, see [8].

Remark 1.4. (Rescaling). For the proof of Theorem 1.1 it is convenient to rescale the discretized torus $\mathbb{T}_{\varepsilon}$ so that the grid size becomes 1 . Recall that $\varepsilon=\frac{1}{L}$ for some integer $L \in \mathbb{N}$. Hence, the $L$-rescaled version of $\mathbb{T}_{\varepsilon}$ yields the discrete rescaled torus $\mathbb{T}_{L}=(\mathbb{Z} / L \mathbb{Z})^{d}=\frac{1}{\varepsilon} \mathbb{T}_{\varepsilon}$. In analogy with (1.8) we introduce discrete derivatives $\nabla$ and $\nabla^{*}$ acting on scalar fields $v: \mathbb{T}_{L} \rightarrow \mathbb{R}$ and vector fields $g=\left(g_{1}, \ldots, g_{d}\right): \mathbb{T}_{L} \rightarrow \mathbb{R}^{d}$ as follows:

$$
\begin{aligned}
& \nabla_{i} v(x)=v\left(x+\mathbf{e}_{i}\right)-v(x), \quad \nabla_{i}^{*} v(x)=v\left(x-\mathbf{e}_{i}\right)-v(x), \\
& \nabla v=\left(\nabla_{1} v, \ldots, \nabla_{d} v\right), \quad \nabla^{*} g=\sum_{i=1}^{d} \nabla_{i}^{*} g_{i} .
\end{aligned}
$$

In order to state Theorem 1.1 in its rescaled version, we set

$$
u(x):=u_{\varepsilon}(\varepsilon x), \quad u_{0}(x):=u_{0, \varepsilon}(\varepsilon x), \quad \tilde{f}(x):=\varepsilon^{2} f_{\varepsilon}(\varepsilon x) \quad \text { for } x \in \mathbb{Z}^{d} .
$$

So defined, $u, u_{0}$ and $\tilde{f}$ are functions on the rescaled torus $\mathbb{T}_{L}$ with vanishing mean, and the finite difference equations (1.9) and (1.12) turn into

$$
\begin{aligned}
\nabla^{*} \boldsymbol{a} \nabla u & =\tilde{f} \text { on } \mathbb{T}_{L}, & & \sum_{\mathbb{T}_{L}} u=0, \\
\nabla^{*} \boldsymbol{a}_{\mathrm{hom}} \nabla u_{0} & =\tilde{f} \text { on } \mathbb{T}_{L}, & & \sum_{\mathbb{T}_{L}} u_{0}=0 .
\end{aligned}
$$


Furthermore, the two-scale expansion in (1.13) takes the form

$$
z:=u-u_{0}-\sum_{j=1}^{d} \phi_{j} \nabla_{j} u_{0}
$$

and estimate (1.13) of Theorem 1.1 can be reformulated as

$$
\left\langle\sum_{\mathbb{T}_{L}}\left(z^{2}+L^{2}|\nabla z|^{2}\right)\right\rangle^{\frac{1}{2}} \lesssim L \mu_{d}^{\frac{1}{2}}(L)\left(\sum_{\mathbb{T}_{L}} \tilde{f}^{2}\right)^{\frac{1}{2}},
$$

where we set for abbreviation

$$
\mu_{d}(L)= \begin{cases}\ln L & \text { for } d=2 \\ 1 & \text { for } d>2\end{cases}
$$

In fact we shall establish (1.19) (and thus (1.13)) in form of the estimate

$$
\left\langle\sum_{\mathbb{T}_{L}}\left(z^{2}+L^{2}|\nabla z|^{2}\right)\right\rangle^{\frac{1}{2}} \lesssim L \mu_{d}^{\frac{1}{2}}(L)\left(\sum_{\mathbb{T}_{L}}\left|\nabla^{2} u_{0}\right|^{2}\right)^{\frac{1}{2}},
$$

where $\nabla^{2} u_{0}(x)$ denotes the discrete Hessian of $u_{0}$ at $x$ and is given by the $d \times d$ matrix with entries $-\nabla_{i}^{*} \nabla_{j} u_{0}(x)$. Note that (1.20) indeed implies (1.19), since $u_{0}$ (as a solution to the constant-coefficient difference equations (1.17)) satisfies the a priori estimate $\sum_{\mathbb{T}_{L}}\left|\nabla^{2} u_{0}\right|^{2} \lesssim \sum_{\mathbb{T}_{L}} f^{2}$ up to a multiplicative constant that only depends on $\lambda$ and $d$.

\section{Notation}

Throughout this article, we use the following notation:

- $d \geq 2$ is the dimension;

- $\left(\mathbf{e}_{1}, \ldots, \mathbf{e}_{d}\right)$ denotes the canonical basis of $\mathbb{Z}^{d}$;

- $\mathbb{T}_{L}=(\mathbb{Z} / L \mathbb{Z})^{d}$ denotes the discretized $L$-rescaled torus;

- $x \bmod L$ denotes the unique point in $([0, L) \cap \mathbb{Z})^{d}$ with $x=(x \bmod L)+L x^{\prime}$ for some $x^{\prime} \in \mathbb{Z}^{d}$;

- $\lesssim$ and $\gtrsim$ stand for $\leq$ and $\geq$ up to a multiplicative constant which only depends on the quantities specified in the context;

- when both $\lesssim$ and $\gtrsim$ hold, we simply write $\sim$;

- $\beta$ denotes a single-site probability measure on $\Omega_{0}$, see Section 2.1 ;

- $\langle\cdot\rangle$ denotes the $L$-periodic i.i.d. ensemble on $\Omega_{L}$ associated with $\beta$, see Section 2.1;

- $\operatorname{cov}[\cdot ; \cdot]$ denotes the covariance associated with $\langle\cdot\rangle$;

- we denote the $(i, j)$ th entry of a $d \times d$-matrix $\boldsymbol{b}$ by $\boldsymbol{b}^{i j}$ and write "." for the inner product in $\mathbb{R}^{d \times d}$, i.e. $\boldsymbol{a}: \boldsymbol{b}=\sum_{i, j=1}^{d} \boldsymbol{a}^{i j} \boldsymbol{b}^{i j}$

- for all $L>0, \mu_{d}(L)=\ln L$ for $d=2$ and $\mu_{d}(L)=1$ for $d>2$.

\section{Assumptions on the ensemble And the notion of the CORRECTor}

In this section we introduce and motivate the required assumptions on the ensemble, the definition of the corrector and the homogenized coefficients. We recall some recent quantitative estimates from stochastic homogenization that are at the basis of the proof of Theorem 1.1. Finally, we comment on the role played by the i.i.d. assumption. 


\subsection{Assumptions on the ensemble}

Recall that $\langle\cdot\rangle$ denotes the i.i.d. ensemble associated with the single-site measure $\beta$ via (1.10). Our theory involves only two probabilistic ingredients: a covariance estimate and a Logarithmic Sobolev Inequality (LSI) for $\langle\cdot\rangle$. We start with the covariance estimate, which is explicitly used in the proof of this paper. We shall comment on the LSI in the following section.

Lemma 2.1. Let $\langle\cdot\rangle$ denote the periodic i.i.d. ensemble (see (1.10)). Then we have for any two random variables $\zeta, \tilde{\zeta}:$

$$
\operatorname{cov}[\zeta ; \tilde{\zeta}] \leq \sum_{y \in \mathbb{T}_{L}}\left\langle\left(\frac{\partial \zeta}{\partial y}\right)^{2}\right\rangle^{\frac{1}{2}}\left\langle\left(\frac{\partial \tilde{\zeta}}{\partial y}\right)^{2}\right\rangle^{\frac{1}{2}}
$$

Here

$$
\operatorname{cov}[\zeta ; \tilde{\zeta}]:=\langle(\zeta-\langle\zeta\rangle)(\tilde{\zeta}-\langle\tilde{\zeta}\rangle)\rangle
$$

denotes the covariance of $\zeta$ and $\tilde{\zeta}$. Furthermore, for a site $y \in \mathbb{T}_{L}$, the random variable $\frac{\partial \zeta}{\partial y}$ is defined by

$$
\left(\frac{\partial \zeta}{\partial y}\right)(\boldsymbol{a}):=\zeta(\boldsymbol{a})-\int_{\Omega_{0}} \zeta(\boldsymbol{a}) \beta(d \boldsymbol{a}(y))
$$

Like the classical partial derivatives $\left\{\frac{\partial \zeta}{\partial \boldsymbol{a}_{k k}(y)}\right\}_{k=1, \ldots, d}$, the function $\frac{\partial \zeta}{\partial y}$ measures how sensitively $\zeta$ depends on the variable $\boldsymbol{a}(y)=\left\{\boldsymbol{a}_{k k}(y)\right\}_{k=1, \ldots, d} \in \Omega_{0}$. For reasons explained in [6] we call these derivatives vertical. For obvious reasons, $\frac{\partial \zeta}{\partial y}$ is called the discrete vertical derivative of $\zeta$ at $y$.

We note that for $\zeta=\tilde{\zeta},(2.1)$ turns into the Spectral Gap Estimate (SG) with constant 1, i.e.

$$
\left\langle\zeta^{2}\right\rangle \leq\left\langle\sum_{y \in \mathbb{T}_{L}}\left(\frac{\partial \zeta}{\partial y}\right)^{2}\right\rangle
$$

for any random variable $\zeta$ with vanishing expectation $\langle\zeta\rangle=0$. We refer to [6], Lemma 2 for a proof in the present context and note that (2.2) is extensively used in the proof of Lemma 2.2 as only probabilistic ingredient. This estimate is reminiscent of the Brascamp-Lieb inequality used by Naddaf and Spencer in [16]. In a slightly different context, a covariance estimate like (2.1) was established in [10], Lemma 3. For the convenience of the reader, we present the elementary proof of Lemma 2.1 .

\subsection{Corrector}

We now introduce the important concept of the corrector. Since we only have to deal with the periodic (as opposed to the infinite) ensemble $\langle\cdot\rangle$, we can avoid discussing all technicalities. Indeed, for any realization of $\boldsymbol{a}$ according to $\langle\cdot\rangle$, that is, for any periodic coefficient field $\boldsymbol{a} \in \Omega_{L}$ and for any coordinate direction $j=1, \ldots, d$, there exists a unique scalar field $\phi_{j}(\boldsymbol{a}, \cdot): \mathbb{T}_{L} \rightarrow \mathbb{R}$ characterized by

$$
\nabla^{*} \boldsymbol{a}\left(\nabla \phi_{j}+\mathbf{e}_{j}\right)=0 \text { on } \mathbb{T}_{L} \text { and } \sum_{\mathbb{T}_{L}} \phi_{j}=0
$$

Here $\mathbf{e}_{j}$ denotes the unit vector in direction of the $j$ th coordinate axis, and the discrete derivatives are defined in (1.15). Clearly, for every $j=1, \ldots, d$, this defines a random scalar field $\phi_{j}$. Evidently, this random field is stationary in the sense that for any shift $y \in \mathbb{R}^{d}$ one has $\phi_{j}(\boldsymbol{a}(\cdot+y), \cdot)=\phi_{j}(\boldsymbol{a}, \cdot+y)$. The periodic function $\phi_{j}: \mathbb{Z}^{d} \rightarrow \mathbb{R}$ "corrects" the affine function $x \mapsto x_{j}$ such that the resulting function $\mathbb{Z}^{d} \ni x \mapsto \phi_{j}(x)+x_{j}$ is $\boldsymbol{a}$-harmonic. In this sense, $\left(\phi_{1}, \ldots, \phi_{d}\right)$ provide $\boldsymbol{a}$-harmonic coordinates for $\mathbb{T}_{L}$. We thus call the $\phi_{j}$ 's the (periodic) corrector. 
A crucial ingredient in the proof of our main result is the following boundedness estimate on the moments of the corrector:

Lemma 2.2 (Gloria, Neukamm \& Otto $[6]$ ). For $j=1, \ldots, d$ we have

$$
\begin{aligned}
\left\langle\left|\phi_{j}\right|^{2}\right\rangle & \lesssim\left\{\begin{array}{ll}
\ln L & \text { for } d=2 \\
1 & \text { for } d>2
\end{array},\right. \\
\left\langle\left|\nabla \phi_{j}\right|^{4}\right\rangle & \lesssim 1 .
\end{aligned}
$$

The multiplicative constants in these estimates only depend on $\lambda$ and $d$.

We remark that the previous estimate indeed holds for the more general class of stationary ensembles which satisfy SG. For the question in which sense $\lim _{L \uparrow \infty} \phi_{j}$ exists, which is however is not relevant for this paper, we refer to [6], Chapter 4.

\subsection{Homogenized coefficient}

Let us recall that a coefficient field

$$
\boldsymbol{a} \in \Omega:=\left\{\boldsymbol{a}: \mathbb{Z}^{d} \rightarrow \Omega_{0}\right\}=\Omega_{0}^{\mathbb{Z}^{d}}
$$

can be seen as a description of a network of resistors: suppose $u: \mathbb{Z}^{d} \rightarrow \mathbb{R}$ is an $\boldsymbol{a}$-harmonic function; if $u(x)$ is interpreted as the potential at vertex $x$ and $\boldsymbol{a}_{k k}(x)$ as conductivity along the edge joining $x$ to $\mathbf{e}_{k}+x$, then $\boldsymbol{a}_{k k}(x) \nabla_{k} u(x)$ can be interpreted as the (stationary) current along this edge. In this sense, the merit of the homogenized coefficient $\boldsymbol{a}_{\text {hom }}$ is that, almost surely, it relates the spatially averaged potential gradient $\xi=\sum \nabla u$ to the spatially averaged current $\boldsymbol{a}_{\mathrm{hom}} \xi=\sum \boldsymbol{a} \nabla u$. For our special $\boldsymbol{a}$-harmonic function $u=\phi_{j}+x_{j}$, the spatially averaged (over one period cell) potential gradient is given by

$$
L^{-d} \sum_{\mathbb{T}_{L}}\left(\nabla \phi_{j}+e_{j}\right)=e_{j},
$$

whereas the spatially averaged current is given by

$$
L^{-d} \sum_{\mathbb{T}_{L}} \boldsymbol{a}\left(\nabla \phi_{j}+e_{j}\right)
$$

Hence the expected value of the latter, that is,

$$
\left\langle L^{-d} \sum_{\mathbb{T}_{L}} \boldsymbol{a}\left(\nabla \phi_{j}+e_{j}\right)\right\rangle \stackrel{\text { stationarity }}{=}\left\langle\boldsymbol{a}(0)\left(\nabla \phi_{j}(0)+e_{j}\right)\right\rangle=: \boldsymbol{a}_{\mathrm{hom}, L} e_{j}
$$

is a good (and computable) proxy for $\boldsymbol{a}_{\mathrm{hom}}$ as $L \uparrow \infty$. Qualitative homogenization theory ensures that it is indeed true that

$$
\lim _{L \uparrow \infty} \boldsymbol{a}_{\mathrm{hom}, L}=\boldsymbol{a}_{\mathrm{hom}},
$$

see for instance [17], Theorem 4.6. For us, this has the convenient side-effect that we do not have to give the technically more demanding, independent definition of $\boldsymbol{a}_{\text {hom }}$. For the latter, we refer to [6], Chapter 5 for instance. Moreover - and this is a second important ingredient for our result - the quantitative theory in [6] provides an optimal estimate of this "systematic error" in the case of the (infinite) i.i.d. ensemble.

Lemma 2.3 (Gloria, Neukamm \& Otto [6], Proposition 1). Consider the i.i.d. ensemble. Then we have

$$
\left|\boldsymbol{a}_{\mathrm{hom}, L}-\boldsymbol{a}_{\mathrm{hom}}\right| \lesssim L^{-d} \ln ^{d} L,
$$

where the multiplicative constant depends only on $d$ and $\lambda$. 
As opposed to the other important ingredients for our result, the annealed estimates on the corrector and on the Green's function in Lemma 2.2 and in Lemma 3.3 below, the above ingredient relies on the i.i.d. property in a subtle way. In fact, if we were not dealing with the i.i.d. ensemble $\langle\cdot\rangle$, but with a more general infinite ensemble (that we call $\langle\cdot\rangle$ for the purpose of the discussion in this paragraph only), the choice of the periodic ensemble $\langle\cdot\rangle_{L}$ (that we endow with the index $L$ for the purpose of this discussion only) is more subtle - and of practical importance: on the one hand, in view of Lemma 2.2 and of Lemma 3.3 below in particular, $\langle\cdot\rangle_{L}$ should satisfy a Logarithmic Sobolev Inequality (LSI) uniformly in $L \uparrow \infty$. On the other hand, in view of Lemma 2.3, $\langle\cdot\rangle_{L}$ should be well-coupled to $\langle\cdot\rangle$ in order to avoid a large systematic error. If the ensemble is not i.i.d., the definition of $\langle\cdot\rangle_{L}$ through a "brutal" periodization, which is obtained by restricting $\boldsymbol{a}(\cdot+y)$ to $\mathbb{T}_{L}$, with a random shift $y \in \mathbb{T}_{L}$ to retain stationarity, seems both unnatural and difficult to control. It seems more natural and promising to us to define $\langle\cdot\rangle_{L}$ as the distribution of $\boldsymbol{a}$ under $\langle\cdot\rangle$ conditioned on the $\mathbb{T}_{L}$-periodicity of $\boldsymbol{a}$. However, this conditioning is singular; and we only expect control if the ensemble $\langle\cdot\rangle$ can be characterized by a sufficiently short-range (translation invariant) Hamiltonian. In this case, we expect that Dobrushin-Shlosman criteria (which as uniform mixing conditions ensure near-independence of $\boldsymbol{a}(x)$ and $\boldsymbol{a}(y)$ for $|x-y| \gg 1$ for all conditional measures) conveniently provide LSI for $\langle\cdot\rangle_{L}$ uniform in $L$. The extension of Lemma 2.3 to such a situation is investigated in a forthcoming work, see [7]. Let us also point out that the present proof relies on the covariance estimate of Lemma 2.1. It is not yet clear to us whether such a covariance estimate can survive beyond the i.i.d. case.

\section{Proofs of Theorem 1.1 And Corollary 1.2}

In this section we present the proofs of Theorem 1.1 and Corollary 1.2. Beforehand, we recall some auxiliary estimates on the elliptic Green's function that we need in the proof.

\section{Structure of the proof and auxiliary estimates on the Green's function}

The starting point to prove Theorem 1.1 is the same as in the proof of [18], Theorem 3 by Papanicolaou and Varadhan. Recall that $z$ is given by (1.18). By uniform ellipticity and $L$-periodicity of $\boldsymbol{a}$, an integration by parts on $\mathbb{T}_{L}$ yields

$$
\lambda\left\langle\sum_{\mathbb{T}_{L}}|\nabla z|^{2}\right\rangle \leq\left\langle\sum_{\mathbb{T}_{L}} \nabla z \cdot \boldsymbol{a} \nabla z\right\rangle=\left\langle\sum_{\mathbb{T}_{L}} z \nabla^{*} \boldsymbol{a} \nabla z\right\rangle .
$$

As we shall see below, in Step 1 of the proof of Theorem 1.1, an application of $\nabla^{*} \boldsymbol{a} \nabla$ to $z$ yields the decomposition

$$
\nabla^{*} a \nabla z=\nabla^{*} g+r_{1}+r_{2}
$$

where the random vector field $g: \mathbb{T}_{L} \rightarrow \mathbb{R}^{d}$, the deterministic scalar field $r_{1}: \mathbb{T}_{L} \rightarrow \mathbb{R}$, and the random scalar field $r_{2}: \mathbb{T}_{L} \rightarrow \mathbb{R}$ are given by

$$
\begin{aligned}
& g_{i}=-\sum_{j=1}^{d} \boldsymbol{a}^{i i} \phi_{j}\left(\cdot+\mathbf{e}_{i}\right) \nabla_{i} \nabla_{j} u_{0}, \\
& r_{1}=\left(\boldsymbol{a}_{\mathrm{hom}, L}-\boldsymbol{a}_{\mathrm{hom}}\right): \nabla^{*} \nabla u_{0}, \\
& r_{2}=\left(\boldsymbol{b}-\boldsymbol{a}_{\mathrm{hom}, L}\right): \nabla^{*} \nabla u_{0} .
\end{aligned}
$$

Above, $\boldsymbol{a}_{\mathrm{hom}, L}$ is defined via (2.6), $\boldsymbol{a}_{\mathrm{hom}}$ is defined via (2.7), and $\boldsymbol{b}: \mathbb{T}_{L} \rightarrow \mathbb{R}^{d \times d}$ denotes the matrix field with entries

$$
\boldsymbol{b}^{i j}:=\boldsymbol{a}^{i i}\left(\cdot-\mathbf{e}_{i}\right)\left(\nabla_{i} \phi_{j}\left(\cdot-\mathbf{e}_{i}\right)+\delta(i-j)\right) \quad \text { for } i, j=1, \ldots, d .
$$

As we shall see below, in Step 3 of the proof of Theorem 1.1, the scalar fields $r_{1}$ and $r_{2}$ satisfy

$$
\sum_{\mathbb{T}_{L}} r_{1}=0 \quad \text { and } \quad\left\langle r_{2}(x)\right\rangle=0 \text { for all } x \in \mathbb{T}_{L} .
$$


By combining (3.1) and (3.2), the bound (1.20) follows from estimates of the terms $\left\langle\sum_{\mathbb{T}_{L}} \nabla z \cdot g\right\rangle$, $\left\langle\sum_{\mathbb{T}_{L}}(z-\bar{z}) r_{1}\right\rangle$ and $\left\langle\sum_{\mathbb{T}_{L}} z r_{2}\right\rangle$, an estimate of the spatial mean

$$
\bar{z}:=L^{-d} \sum_{\mathbb{T}_{L}} z
$$

and the discrete Poincaré inequality on the torus.

The most intricate estimate is the one of $\left\langle\sum_{\mathbb{T}_{L}} z r_{2}\right\rangle$, see Step 5 in the Proof of Theorem 1.1 below. Since the term $r_{2}(x)$ has vanishing expectation for all $x \in \mathbb{T}_{L}$ one may write it as a covariance

$$
\left\langle\sum_{x \in \mathbb{T}_{L}} z(x) r_{2}(x)\right\rangle=\sum_{x \in \mathbb{T}_{L}} \operatorname{cov}\left[z(x) ; r_{2}(x)\right] .
$$

In order to benefit from this, we appeal to the covariance estimate of Lemma 2.1 and to a vertical derivative calculus on coefficient fields that we introduced in [6]. The process of estimating the vertical derivatives of $r_{2}$ and $z$ involves the (periodic) Green's function:

Definition 3.1. The $L$-periodic Green's function $G_{L}: \mathbb{T}_{L} \times \mathbb{T}_{L} \times \Omega_{L} \rightarrow \mathbb{R}$ is defined as follows. For all $y \in \mathbb{T}_{L}$ and $\boldsymbol{a} \in \Omega_{L}$ the function $G_{L}(\cdot, y ; \boldsymbol{a})$ is the unique $L$-periodic mean free solution to

$$
\nabla^{*} \boldsymbol{a} \nabla G_{L}(\cdot, y ; \boldsymbol{a})=\delta(\cdot-y)-L^{-d} \quad \text { on } \mathbb{T}_{L},
$$

where $\delta$ is the Dirac mass at zero.

When no confusion occurs we use the shorthand notation $G_{L}(x, y)$ for $G_{L}(x, y ; \boldsymbol{a})$. We shall use both quenched (i.e. pointwise deterministic) and annealed (i.e. statistically averaged) estimates on $\left|\nabla G_{L}\right|$. The pointwise estimates rely on the De Giorgi-Nash-Moser Hölder regularity theory (and are standard in the continuum case):

Lemma 3.2. There exists $\gamma>0$ depending only on $\lambda$ and $d$ such that for all $\boldsymbol{a} \in \Omega_{L}$ and $L \in \mathbb{N}$, the Green's function $G_{L}(\cdot, \cdot ; \boldsymbol{a})$ satisfies the following quenched estimate:

$$
\left|\nabla_{x} G_{L}(x, y ; \boldsymbol{a})\right|,\left|\nabla_{y} G_{L}(x, y ; \boldsymbol{a})\right| \lesssim(|x-y \bmod L|+1)^{2-d-\gamma} .
$$

In the estimate the multiplicative constant only depends on $\lambda$ and $d$. (Note that $G_{L}$ is symmetric, so that the estimate (3.7) does not depend on the variable with respect to which we differentiate.)

See Appendix A for the proof. The crucial other ingredient is the recent annealed estimate of [14] by Marahrens and the third author, which we recall below in a version for the $L$-periodic Green's functions:

Lemma 3.3 (Marahrens \& Otto [14], Theorem 1). The periodic Green's function $G_{L}$ satisfies the following annealed estimates:

$$
\left\langle\left|\nabla G_{L}(x, y)\right|^{4}\right\rangle^{\frac{1}{4}} \lesssim(|x-y \bmod L|+1)^{1-d},
$$

and

$$
\left\langle\left|\nabla_{x} \nabla_{y} G_{L}(x, y)\right|^{4}\right\rangle^{\frac{1}{4}} \lesssim(|x-y \bmod L|+1)^{-d},
$$

where the multiplicative constants only depend on $\lambda$ and $d$.

Let us mention that the proof of the annealed estimates on the derivatives of the Green's functions relies on a strengthened version of the spectral gap estimate (2.2), namely a Logarithmic-Sobolev Inequality. We refer the reader to [14] for details. Note that as for the variance estimate by Naddaf and Spencer in [16], an optimal control of the fourth moment is enough for our quantitative expansion. 


\section{Proof of Theorem 1.1}

We prove the estimate of Theorem 1.1 in its rescaled formulation (1.20). Note that the identity

$$
\sum_{\mathbb{T}_{L}} \sum_{i, j=1}^{d}\left|\nabla_{i}^{*} \nabla_{j} u_{0}\right|^{2}=\sum_{\mathbb{T}_{L}} \sum_{i, j=1}^{d}\left|\nabla_{i} \nabla_{j} u_{0}\right|^{2}=\sum_{\mathbb{T}_{L}}\left|\nabla^{2} u_{0}\right|^{2}
$$

follows from periodicity and the elementary identity $\left|\nabla_{i}^{*} \nabla_{j} u_{0}(\cdot)\right|=\left|\nabla_{i} \nabla_{j} u_{0}\left(\cdot-\mathbf{e}_{i}\right)\right|$. The argument is divided into five steps. In the first step we derive the decomposition (3.2) with property (3.5). In Steps 2 and 3 we argue that it suffices to prove

$$
\left\langle\sum_{\mathbb{T}_{L}}|\nabla z|^{2}\right\rangle \lesssim \mu_{d}(L) \sum_{\mathbb{T}_{L}}\left|\nabla^{2} u_{0}\right|^{2}
$$

In the remaining steps we prove (3.11) starting with the inequality $\lambda\left\langle\sum_{\mathbb{T}_{L}}|\nabla z|^{2}\right\rangle \leq\left\langle\sum_{\mathbb{T}_{L}} z \nabla^{*} \boldsymbol{a} \nabla z\right\rangle$. To that end we appeal to the representation formula (3.2) which is a sum of a term in divergence form $\nabla^{*} g$, a deterministic term $r_{1}$ and a remainder with vanishing expectation $r_{2}$. The first two terms are estimated in Step 4 . The third term $r_{2}$, which has vanishing expectation, is controlled using the covariance estimate of Lemma 2.1 and will be treated in Steps $5 \mathrm{a}-5 \mathrm{~d}$.

Step 1. Derivation of the decomposition (3.2) with property (3.5).

Let us show that (3.2) holds with $g, r_{1}$ and $r_{2}$ given by (3.3). By the defining equation (1.9) for $u$,

$$
\begin{gathered}
\nabla^{*} \boldsymbol{a} \nabla z \stackrel{(1.18)}{=} \nabla^{*} \boldsymbol{a} \nabla u-\nabla^{*} \boldsymbol{a} \nabla u_{0}-\sum_{j=1}^{d} \nabla^{*} \boldsymbol{a} \nabla\left(\phi_{j} \nabla_{j} u_{0}\right) \\
\stackrel{(1.9)}{=} \tilde{f}-\underbrace{\nabla^{*} \boldsymbol{a} \nabla u_{0}}_{=: I}-\underbrace{\sum_{j=1}^{d} \nabla^{*} \boldsymbol{a} \nabla\left(\phi_{j} \nabla_{j} u_{0}\right)}_{=: I I} .
\end{gathered}
$$

We shall use the following discrete Leibniz rule: for all $\zeta_{1}, \zeta_{2}: \mathbb{T}_{L} \rightarrow \mathbb{R}$,

$$
\nabla_{i}\left(\zeta_{1} \zeta_{2}\right)=\left(\nabla_{i} \zeta_{1}\right) \zeta_{2}+\zeta_{1}\left(\cdot+\mathbf{e}_{i}\right) \nabla_{i} \zeta_{2}, \quad \nabla_{i}^{*}\left(\zeta_{1} \zeta_{2}\right)=\left(\nabla_{i}^{*} \zeta_{1}\right) \zeta_{2}+\zeta_{1}\left(\cdot-\mathbf{e}_{i}\right) \nabla_{i}^{*} \zeta_{2} .
$$

For the first term this yields

$$
I=\sum_{i=1}^{d}\left(\nabla_{i}^{*} \boldsymbol{a}^{i i}\right) \nabla_{i} u_{0}+\sum_{i=1}^{d} \boldsymbol{a}^{i i}\left(\cdot-\mathbf{e}_{i}\right) \nabla_{i}^{*} \nabla_{i} u_{0},
$$

while for the second term we obtain

$$
\begin{aligned}
& I I=\sum_{i, j=1}^{d} \nabla_{i}^{*}\left(\boldsymbol{a}^{i i} \nabla_{i}\left(\phi_{j} \nabla_{j} u_{0}\right)\right) \\
& \stackrel{(3.13)}{=} \sum_{i, j=1}^{d} \nabla_{i}^{*}\left(\boldsymbol{a}^{i i}\left(\nabla_{i} \phi_{j} \nabla_{j} u_{0}+\phi_{j}\left(\cdot+\mathbf{e}_{i}\right) \nabla_{i} \nabla_{j} u_{0}\right)\right) \\
& \stackrel{(3.13)}{=} \sum_{i, j=1}^{d} \nabla_{i}^{*}\left(\boldsymbol{a}^{i i} \nabla_{i} \phi_{j}\right) \nabla_{j} u_{0}+\sum_{i, j=1}^{d} \boldsymbol{a}^{i i}\left(\cdot-\mathbf{e}_{i}\right) \nabla_{i} \phi_{j}\left(\cdot-\mathbf{e}_{i}\right) \nabla_{i}^{*} \nabla_{j} u_{0} \\
& \quad+\sum_{i, j=1}^{d} \nabla_{i}^{*}\left(\boldsymbol{a}^{i i} \phi_{j}\left(\cdot+\mathbf{e}_{i}\right) \nabla_{i} \nabla_{j} u_{0}\right) .
\end{aligned}
$$


Because of the periodic corrector equation (2.3), the first term of the r.h.s. turns into

$$
\sum_{i, j=1}^{d} \nabla_{i}^{*}\left(\boldsymbol{a}^{i i} \nabla_{i} \phi_{j}\right) \nabla_{j} u_{0}=-\sum_{j=1}^{d}\left(\nabla_{j}^{*} \boldsymbol{a}^{j j}\right) \nabla_{j} u_{0}
$$

Hence, the terms in $I+I I$ that involve the first derivative of $u_{0}$ cancel, so that

$$
\begin{aligned}
I+I I= & \sum_{i, j=1}^{d} \boldsymbol{a}^{i i}\left(\cdot-\mathbf{e}_{i}\right)\left(\delta(i-j)+\nabla_{i} \phi_{j}\left(\cdot-\mathbf{e}_{i}\right)\right) \nabla_{i}^{*} \nabla_{j} u_{0} \\
& +\sum_{i, j=1}^{d} \nabla_{i}^{*}\left(\boldsymbol{a}^{i i} \phi_{j}\left(\cdot+\mathbf{e}_{i}\right) \nabla_{i} \nabla_{j} u_{0}\right) .
\end{aligned}
$$

The last term on the r.h.s. is precisely $-\nabla^{*} g$, the first term is $\boldsymbol{b}: \nabla^{2} u_{0}$, cf. (3.3) and (3.4). The claim (3.2) then follows from (3.12), (3.14) and identity (1.17) which can be written in the form $\tilde{f}=\boldsymbol{a}_{\text {hom }}: \nabla^{*} \nabla u_{0}$ since $\boldsymbol{a}_{\text {hom }}$ is constant.

To conclude this step, we prove (3.5). The first identity simply follows from the $L$-periodicity of $u_{0}$. The second identity can be seen as follows: By the definition of $\boldsymbol{b}$, the stationarity of $\boldsymbol{a}$ and $\phi_{j}$, and the definition of $\boldsymbol{a}_{\text {hom }, L}$ via (2.6) we have

$$
\left\langle\boldsymbol{b}^{i j}(\cdot)\right\rangle=\left\langle\boldsymbol{a}^{i i}(0)\left(\delta(i-j)+\nabla_{i} \phi_{j}(0)\right)\right\rangle=\boldsymbol{a}_{\mathrm{hom}, L}^{i j},
$$

so that

$$
\left\langle\boldsymbol{b}: \nabla^{2} u_{0}\right\rangle=\langle\boldsymbol{b}\rangle: \nabla^{2} u_{0}=\boldsymbol{a}_{\mathrm{hom}, L}: \nabla^{2} u_{0}
$$

as desired.

Step 2. Reduction to an estimate for $\nabla z$.

We claim that (1.20) and thus the statement of Theorem 1.1, see Remark 1.4, follows from (3.11). Indeed, by the discrete Poincaré inequality $\sum_{\mathbb{T}_{L}} z^{2} \lesssim L^{2} \sum_{\mathbb{T}_{L}}|\nabla z|^{2}+L^{d} \bar{z}^{2}$ we only need to prove that

$$
\left\langle\bar{z}^{2}\right\rangle \lesssim L^{2-d} \mu_{d}(L) \sum_{\mathbb{T}_{L}}\left|\nabla^{2} u_{0}\right|^{2}
$$

Since the spatial means of $u$ and $u_{0}$ vanish by definition, we have $\bar{z}=-L^{-d} \sum_{\mathbb{T}_{L}} \sum_{j=1}^{d} \phi_{j} \nabla_{j} u_{0}$, so that

$$
\left\langle\bar{z}^{2}\right\rangle=L^{-2 d} \sum_{x \in \mathbb{T}_{L}} \sum_{x^{\prime} \in \mathbb{T}_{L}}\left\langle\left(\sum_{i=1}^{d} \phi_{i}(x) \nabla_{i} u_{0}(x)\right)\left(\sum_{j=1}^{d} \phi_{j}\left(x^{\prime}\right) \nabla_{j} u_{0}\left(x^{\prime}\right)\right)\right\rangle \text {. }
$$

We expand the square on the r.h.s. Since $u_{0}$ is deterministic we get

$$
\left\langle\bar{z}^{2}\right\rangle=L^{-2 d} \sum_{x \in \mathbb{T}_{L}} \sum_{x^{\prime} \in \mathbb{T}_{L}} \sum_{i, j=1}^{d} \nabla_{i} u_{0}(x) \nabla_{j} u_{0}\left(x^{\prime}\right)\left\langle\phi_{i}(x) \phi_{j}\left(x^{\prime}\right)\right\rangle .
$$

By Cauchy-Schwarz' inequality, stationarity of the correctors, and the bounds of Lemma 2.2,

$$
\left\langle\phi_{i}(x) \phi_{j}\left(x^{\prime}\right)\right\rangle \leq \max _{k=1, \ldots, d}\left\langle\phi_{k}^{2}\right\rangle \lesssim \mu_{d}(L) .
$$


Hence,

$$
\left\langle\bar{z}^{2}\right\rangle \lesssim \mu_{d}(L)\left(L^{-d} \sum_{x \in \mathbb{T}_{L}}\left|\nabla u_{0}\right|\right)^{2}
$$

The desired estimate then follows from Jensen's and Poincaré's inequalities.

Step 3. Reduction based on the decomposition (3.2).

In this step we argue that the desired estimate reduces to the following statement: suppose that the functions $g$, $r_{1}$ and $r_{2}$ of the decompositon (3.2) satisfy the following estimates in addition to (3.5):

$$
\begin{aligned}
\left\langle\sum_{\mathbb{T}_{L}}|g|^{2}\right\rangle & \lesssim \mu_{d}(L) \sum_{\mathbb{T}_{L}}\left|\nabla^{2} u_{0}\right|^{2}, \\
\sum_{\mathbb{T}_{L}} r_{1}^{2} & \lesssim \frac{\ln ^{d} L}{L^{d}} \sum_{\mathbb{T}_{L}}\left|\nabla^{2} u_{0}\right|^{2}, \\
\left\langle\sum_{\mathbb{T}_{L}} z r_{2}\right\rangle & \lesssim\left\langle\sum_{\mathbb{T}_{L}}|\nabla z|^{2}\right\rangle^{\frac{1}{2}}\left(\sum_{\mathbb{T}_{L}}\left|\nabla^{2} u_{0}\right|^{2}\right)^{\frac{1}{2}}+\mu_{d}(L) \sum_{\mathbb{T}_{L}}\left|\nabla^{2} u_{0}\right|^{2},
\end{aligned}
$$

then estimate (1.20) and thus Theorem 1.1, holds.

By Step 2 we just have to check (3.11). Indeed, by combining (3.1), the decomposition (3.2), the triangle inequality, an integration by parts, and (3.5) we get

$$
\lambda\left\langle\sum_{\mathbb{T}_{L}}|\nabla z|^{2}\right\rangle \leq\left|\left\langle\sum_{\mathbb{T}_{L}} \nabla z \cdot g\right\rangle\right|+\left|\left\langle\sum_{\mathbb{T}_{L}}(z-\bar{z}) r_{1}\right\rangle\right|+\left|\left\langle\sum_{\mathbb{T}_{L}} z r_{2}\right\rangle\right| .
$$

The first term is estimated by (3.15) and the Cauchy-Schwarz inequality:

$$
\left|\left\langle\sum_{\mathbb{T}_{L}} \nabla z \cdot g\right\rangle\right| \lesssim \mu_{d}^{\frac{1}{2}}(L)\left\langle\sum_{\mathbb{T}_{L}}|\nabla z|^{2}\right\rangle^{\frac{1}{2}}\left(\sum_{\mathbb{T}_{L}}\left|\nabla^{2} u_{0}\right|^{2}\right)^{\frac{1}{2}}
$$

The second term is estimated by (3.16), the Cauchy-Schwarz inequality, the Poincaré inequality for functions on $\mathbb{T}_{L}$ with zero mean, and the elementary estimate $\frac{\ln ^{d} L}{L^{d-1}} \lesssim 1$ for $d>1$ :

$$
\left|\left\langle\sum_{\mathbb{T}_{L}}(z-\bar{z}) r_{1}\right\rangle\right| \lesssim\left\langle\sum_{\mathbb{T}_{L}}|\nabla z|^{2}\right\rangle^{\frac{1}{2}}\left(\sum_{\mathbb{T}_{L}}\left|\nabla^{2} u_{0}\right|^{2}\right)^{\frac{1}{2}} .
$$

The combination of (3.17) with the previous three inequalities yields

$$
\left\langle\sum_{\mathbb{T}_{L}}|\nabla z|^{2}\right\rangle \lesssim \mu_{d}^{\frac{1}{2}}(L)\left\langle\sum_{\mathbb{T}_{L}}|\nabla z|^{2}\right\rangle^{\frac{1}{2}}\left(\sum_{\mathbb{T}_{L}}\left|\nabla^{2} u_{0}\right|^{2}\right)^{\frac{1}{2}}+\mu_{d}(L) \sum_{\mathbb{T}_{L}}\left|\nabla^{2} u_{0}\right|^{2},
$$

which implies (3.11) by Young's inequality.

Step 4. Proof of the estimates (3.15) and (3.16).

Estimate (3.15) follows from the definition of $g$, cf. (3.3), the bound (2.4) of Lemma 2.2 on the second moment of the stationary $\phi_{j}$ and identity (3.10). Similarly, (3.16) follows from the definition of $r_{1}$ and the optimal bound on the error $\left|\boldsymbol{a}_{\mathrm{hom}, L}-\boldsymbol{a}_{\mathrm{hom}}\right|$ of Lemma 2.3 . 
In the last step we prove (3.17). Since this step is rather long, we subdivide it further.

Step 5a. Application of the covariance estimate.

Since by $(3.5),\left\langle r_{2}(x)\right\rangle=0$ for all $x \in \mathbb{T}_{L}$, we have

$$
\begin{aligned}
& \left|\left\langle\sum_{x \in \mathbb{T}_{L}} z(x) r_{2}(x)\right\rangle\right|=\left|\sum_{x \in \mathbb{T}_{L}} \operatorname{cov}\left[z(x) ; r_{2}(x)\right]\right| \\
& \stackrel{(3.3)}{=}\left|\sum_{x \in \mathbb{T}_{L}} \sum_{i, j=1}^{d} \nabla_{i}^{*} \nabla_{j} u_{0}(x) \operatorname{cov}\left[z(x) ; \boldsymbol{a}_{\mathrm{hom}, L}^{i j}-\boldsymbol{b}^{i j}(x)\right]\right| \\
& \quad \stackrel{(3.10)}{\leq}\left(\sum_{\mathbb{T}_{L}}\left|\nabla^{2} u_{0}\right|^{2}\right)^{\frac{1}{2}}\left(\sum_{x \in \mathbb{T}_{L}} \sum_{i, j=1}^{d}\left(\operatorname{cov}\left[z(x) ; \boldsymbol{a}_{\mathrm{hom}, L}^{i j}-\boldsymbol{b}^{i j}(x)\right]\right)^{2}\right)^{\frac{1}{2}} .
\end{aligned}
$$

With the covariance estimate in Lemma 2.1 the r.h.s. is bounded by

$$
\left(\sum_{\mathbb{T}_{L}}\left|\nabla^{2} u_{0}\right|^{2}\right)^{\frac{1}{2}}\left(\sum_{x \in \mathbb{T}_{L}} \sum_{i, j=1}^{d}\left(\sum_{y \in \mathbb{T}_{L}}\left\langle\left(\frac{\partial z(x)}{\partial y}\right)^{2}\right\rangle^{\frac{1}{2}}\left\langle\left(\frac{\partial \boldsymbol{b}^{i j}(x)}{\partial y}\right)^{2}\right\rangle^{\frac{1}{2}}\right)^{2}\right)^{\frac{1}{2}} .
$$

Hence, for (3.17) it suffices to prove that

$$
\begin{aligned}
& \left(\sum_{x \in \mathbb{T}_{L}}\left\langle\sum_{y \in \mathbb{T}_{L}}\left\langle\left(\frac{\partial z(x)}{\partial y}\right)^{2}\right\rangle^{\frac{1}{2}}\left\langle\left(\frac{\partial \boldsymbol{b}^{i j}(x)}{\partial y}\right)^{2}\right\rangle^{\frac{1}{2}}\right)^{2}\right)^{\frac{1}{2}} \\
& \leq\left\langle\sum_{\mathbb{T}_{L}}|\nabla z|^{2}\right\rangle^{\frac{1}{2}}+\mu_{d}(L)\left(\sum_{\mathbb{T}_{L}}\left|\nabla^{2} u_{0}\right|^{2}\right)^{\frac{1}{2}} \quad \text { for } i, j=1, \ldots, d .
\end{aligned}
$$

To estimate the vertical derivatives we need to identify $\frac{\partial z}{\partial y}$ and $\frac{\partial \boldsymbol{b}^{i j}}{\partial y}$. This is done by appealing to the elliptic equations (1.16) and (2.3) and vertical differential calculus. Since the basic argument is simple, but polluted due to the discrete nature of the vertical and spatial derivatives, we will first present a formal calculation where the vertical derivative $\frac{\partial}{\partial y}$ is replaced by the classical partial derivative $\frac{\partial}{\partial \boldsymbol{a}_{k k}(y)}$ (defined for differentiable functions on $\Omega_{L}$ ). The rigorous argument is then carried out in Steps $5 \mathrm{c}$ and $5 \mathrm{~d}$ below.

Step 5b. Formal derivation of formulas for the vertical derivatives.

We first (formally) identify $\frac{\partial u}{\partial \boldsymbol{a}_{k k}(y)}$ and $\frac{\partial \phi_{i}(x)}{\partial \boldsymbol{a}(x)}$. Applying $\frac{\partial}{\partial \boldsymbol{a}_{k k}(y)}$ to the elliptic equations (1.16) and (2.3) yields

$$
\begin{aligned}
& \nabla^{*} \boldsymbol{a}(x) \nabla \frac{\partial u(x)}{\partial \boldsymbol{a}_{k k}(y)}=-\nabla^{*} \frac{\partial \boldsymbol{a}(x)}{\partial \boldsymbol{a}_{k k}(y)} \nabla u(x), \\
& \nabla^{*} \boldsymbol{a}(x) \nabla \frac{\partial \phi_{j}(x)}{\partial \boldsymbol{a}_{k k}(y)}=-\nabla^{*} \frac{\partial \boldsymbol{a}(x)}{\partial \boldsymbol{a}_{k k}(y)}\left(\nabla \phi_{j}(x)+\mathbf{e}_{j}\right),
\end{aligned}
$$


using that $\frac{\partial}{\partial \boldsymbol{a}_{k k}(y)}$ and $\nabla$ commute. Since $\frac{\partial \boldsymbol{a}(x)}{\partial \boldsymbol{a}_{k k}(y)}=\left(\mathbf{e}_{k} \otimes \mathbf{e}_{k}\right) \delta(x-y \bmod L)$ for all $x, y \in \mathbb{T}_{L}$, the Green representation formula yields

$$
\begin{aligned}
& \frac{\partial u(x)}{\partial \boldsymbol{a}_{k k}(y)}=-\nabla_{y_{k}} G_{L}(x, y) \nabla_{k} u(y), \\
& \frac{\partial \phi_{j}(x)}{\partial \boldsymbol{a}_{k k}(y)}=-\nabla_{y_{k}} G_{L}(x, y)\left(\nabla_{k} \phi_{j}(y)+\delta(k-j)\right) .
\end{aligned}
$$

Next we identify $\frac{\partial \boldsymbol{b}^{i j}}{\partial \boldsymbol{a}_{k k}(y)}$. We apply $\frac{\partial}{\partial \boldsymbol{a}_{k k}(y)}$ to the definition (3.4) of $\boldsymbol{b}^{i j}$ and use the identity above in the form of

$$
\frac{\partial \nabla_{i} \phi_{j}(x)}{\partial \boldsymbol{a}_{k k}(y)}=-\nabla_{x_{i}} \nabla_{y_{k}} G_{L}(x, y)\left(\nabla_{k} \phi_{j}(y)+\delta(k-j)\right) .
$$

Rearranging the terms yields the identity

$$
\begin{aligned}
\frac{\partial \boldsymbol{b}^{i j}\left(x+\mathbf{e}_{i}\right)}{\partial \boldsymbol{a}_{k k}(y)}= & \delta(k-i) \delta(x-y \bmod L)\left(\nabla_{i} \phi_{j}(y)+\delta(i-j)\right) \\
& -\boldsymbol{a}^{i i}(x) \nabla_{x_{i}} \nabla_{y_{k}} G_{L}(x, y)\left(\nabla_{k} \phi_{j}(y)+\delta(k-j)\right) .
\end{aligned}
$$

Likewise, for the identification of $\frac{\partial z(x)}{\partial \boldsymbol{a}_{k k}(y)}$ we apply $\frac{\partial}{\partial \boldsymbol{a}_{k k}(y)}$ to (1.18):

$$
\begin{aligned}
\frac{\partial z(x)}{\partial \boldsymbol{a}_{k k}(y)} & =\frac{\partial u(x)}{\partial \boldsymbol{a}_{k k}(y)}-\sum_{j=1}^{d} \frac{\partial \phi_{j}(x)}{\partial \boldsymbol{a}_{k k}(y)} \nabla_{j} u_{0}(x) \\
& \stackrel{(3.19)}{=}-\nabla_{y_{k}} G_{L}(x, y)\left(\nabla_{k} u(y)-\sum_{j=1}^{d}\left(\nabla_{k} \phi_{j}(y)+\delta(k-j)\right) \nabla_{j} u_{0}(x)\right) .
\end{aligned}
$$

Since we want to make $\nabla z$ appear, we substitute $\nabla u$ by the following expression

$$
\nabla u(y)=\nabla z(y)+\sum_{j=1}^{d}\left(\nabla \phi_{j}(y)+\mathbf{e}_{j}\right) \nabla_{j} u_{0}(y)+\sum_{j=1}^{d} \phi_{j}(y) \nabla \nabla_{j} u_{0}(y),
$$

which can formally be obtained by applying $\nabla$ to (1.18) and using the continuum Leibniz rule $\nabla\left(\phi_{j} \nabla_{j} u_{0}\right)=$ $\nabla \phi_{j} \nabla_{j} u_{0}+\phi_{j} \nabla \nabla_{j} u$. We then get

$$
\begin{aligned}
\frac{\partial z(x)}{\partial \boldsymbol{a}_{k k}(y)}=- & \nabla_{y_{k}} G_{L}(x, y)\left(\nabla_{k} z(y)+\sum_{j=1}^{d}\left(\nabla_{k} \phi_{j}(y)+\delta(k-j)\right)\left(\nabla_{j} u_{0}(y)-\nabla_{j} u_{0}(x)\right)\right. \\
& \left.+\sum_{j=1}^{d} \phi_{j}(y) \nabla_{k} \nabla_{j} u_{0}(y)\right) .
\end{aligned}
$$

Let us stress the fact that the expression in the brackets on the r.h.s. is $\nabla z(y)$ plus terms that vanish if $u_{0}$ is affine. This will be crucial in order to obtain an optimal estimate.

Step 5c. Rigorous derivation of formulas for the vertical derivatives.

We now derive rigorous versions of (3.21) and (3.23), which will lead to the desired estimate (3.18). For the rigorous argument $\frac{\partial}{\partial \boldsymbol{a}_{k k}(y)}$ has to be replaced by the discrete vertical derivative $\frac{\partial}{\partial y}$ for which the Leibniz rule 
is not valid. The main ingredient is the following rigorous version of (3.19): For $j=1, \ldots, d$ and $x, y \in \mathbb{T}_{L}$ we have

$$
\begin{aligned}
\frac{\partial \phi_{j}(x)}{\partial y} & =-\nabla_{y} G_{L}(x, y) \cdot\left[\nabla \phi_{j}(y)+\mathbf{e}_{j}\right]_{y}, \\
\frac{\partial \nabla_{i} \phi_{j}(x)}{\partial y} & =-\nabla_{x_{i}} \nabla_{y} G_{L}(x, y) \cdot\left[\nabla \phi_{j}(y)+\mathbf{e}_{j}\right]_{y} \\
\frac{\partial u(x)}{\partial y} & =-\nabla_{y} G_{L}(x, y) \cdot[\nabla u(y)]_{y},
\end{aligned}
$$

where $[\cdot]_{y}$ denotes the commutator of the multiplication with $\boldsymbol{a}(y)$ and $\frac{\partial}{\partial y}$, i.e.

$$
[F]_{y}:=\frac{\partial(\boldsymbol{a}(y) F)}{\partial y}-\boldsymbol{a}(y) \frac{\partial F}{\partial y}=\boldsymbol{a}(y)\langle F\rangle_{y}-\langle\boldsymbol{a}(y) F\rangle_{y}
$$

for all random vectors $F$. By Jensen's inequality, the commutator satisfies the following estimate: For all $1 \leq$ $q<\infty$,

$$
\left\langle\left|[F]_{y}\right|^{q}\right\rangle \leq 2^{q}\left\langle|F|^{q}\right\rangle,
$$

which we will use in the sequel for $q=2$ and $q=4$. Note that for all $x, y \in \mathbb{T}_{L}$, since the coefficients are i.i.d.,

$$
\frac{\partial(\boldsymbol{a}(x) F)}{\partial y}-\boldsymbol{a}(x) \frac{\partial F}{\partial y}=[F]_{y} \delta(x-y \bmod L) .
$$

Here comes the argument for (3.24). By (2.3) we have

$$
\begin{aligned}
0 & =\nabla^{*} \boldsymbol{a}(x)\left(\nabla \phi_{j}(x)+\mathbf{e}_{j}\right)-\left\langle\nabla^{*} \boldsymbol{a}(x)\left(\nabla \phi_{j}(x)+\mathbf{e}_{j}\right)\right\rangle_{y} \\
& =\nabla^{*}\left(\boldsymbol{a}(x)\left(\nabla \phi_{j}(x)+\mathbf{e}_{j}\right)-\left\langle\boldsymbol{a}(x)\left(\nabla \phi_{j}(x)+\mathbf{e}_{j}\right)\right\rangle_{y}\right) \\
& =\nabla^{*}\left(\boldsymbol{a}(x) \frac{\partial \nabla \phi_{j}(x)}{\partial y}+\boldsymbol{a}(x)\left\langle\nabla \phi_{j}(x)+\mathbf{e}_{j}\right\rangle_{y}-\left\langle\boldsymbol{a}(x)\left(\nabla \phi_{j}(x)+\mathbf{e}_{j}\right)\right\rangle_{y}\right)
\end{aligned}
$$

Using then (3.27) and (3.29) for $F=\nabla \phi_{j}(x)+\mathbf{e}_{j}$, this turns into

$$
\nabla^{*}\left(\boldsymbol{a}(x) \nabla \frac{\partial \phi_{j}(x)}{\partial y}\right)=-\nabla^{*}\left(\left[\nabla \phi_{j}(x)+\mathbf{e}_{j}\right]_{y} \delta(x-y \bmod L)\right),
$$

from which (3.24) follows by the Green representation formula and an integration by parts. Identity (3.25) follows from applying $\nabla_{x}$ to (3.24). The argument for (3.26) is similar to the one for (3.24) and left to the reader.

We are now in position to derive the rigorous versions of (3.21) and (3.23), and start with $b^{i j}$. We claim that

$$
\frac{\partial \boldsymbol{b}^{i j}\left(x+\mathbf{e}_{i}\right)}{\partial y}=\left(\delta(x-y \bmod L) \mathbf{e}_{i}-\boldsymbol{a}^{i i}(x) \nabla_{x_{i}} \nabla_{y} G_{L}(x, y)\right) \cdot\left[\nabla \phi_{j}(y)+\mathbf{e}_{j}\right]_{y},
$$

Indeed, using again (3.27) for $F=\nabla \phi_{j}(x)+\mathbf{e}_{j}$, we have

$$
\begin{aligned}
\frac{\partial\left(\boldsymbol{a}(x)\left(\nabla \phi_{j}(x)+\mathbf{e}_{j}\right)\right)}{\partial y} & =\boldsymbol{a}(x) \nabla \frac{\partial \phi_{j}(x)}{\partial y}+\boldsymbol{a}(x)\left\langle\nabla \phi_{j}(x)+\mathbf{e}_{j}\right\rangle_{y}-\left\langle\boldsymbol{a}(x)\left(\nabla \phi_{j}(x)+\mathbf{e}_{j}\right)\right\rangle_{y} \\
& =\boldsymbol{a}(x) \frac{\partial \nabla \phi_{j}(x)}{\partial y}+\delta(x-y \bmod L)\left[\nabla \phi_{j}(y)+\mathbf{e}_{j}\right]_{y},
\end{aligned}
$$

which, combined with (3.25), yields (3.30). 
We then turn to $z$ and claim that

$$
\frac{\partial z(x)}{\partial y}=-\nabla_{y} G_{L}(x, y) \cdot\left(F_{1}(y)+F_{2}(y, x)+F_{3}(y)\right)
$$

where

$$
\begin{aligned}
& F_{1}(y):=[\nabla z(y)]_{y}, \quad F_{2}(y, x):=\sum_{j=1}^{d}\left(\nabla_{j} u_{0}(y)-\nabla_{j} u_{0}(x)\right)\left[\nabla \phi_{j}(y)+\mathbf{e}_{j}\right]_{y}, \\
& F_{3}(y):=\sum_{i, j=1}^{d} \nabla_{i} \nabla_{j} u_{0}(y)\left[\phi_{j}\left(y+\mathbf{e}_{i}\right) \mathbf{e}_{i}\right]_{y} .
\end{aligned}
$$

We first apply $\frac{\partial}{\partial y}$ to (1.18) and use (3.24) and (3.26):

$$
\frac{\partial z(x)}{\partial y}=-\nabla_{y} G_{L}(x, y) \cdot\left([\nabla u(y)]_{y}-\sum_{j=1}^{d} \nabla_{j} u_{0}(x)\left[\nabla \phi_{j}(y)+\mathbf{e}_{j}\right]_{y}\right) .
$$

We then wish to substitute the term $\nabla u(y)$ by an expression that involves $\nabla z(y)$. To that end we apply $\nabla$ to (1.18) and get with the help of the discrete Leibniz rule (3.13)

$$
\begin{aligned}
\nabla u(y) & =\nabla z(y)+\nabla u_{0}(y)+\sum_{j=1}^{d} \nabla_{j} u_{0}(y) \nabla \phi_{j}(y)+\sum_{i, j=1}^{d} \nabla_{i} \nabla_{j} u_{0}(y) \phi_{j}\left(y+\mathbf{e}_{i}\right) \mathbf{e}_{i} \\
& =\nabla z(y)+\sum_{j=1}^{d} \nabla_{j} u_{0}(y)\left(\nabla \phi_{j}(y)+\mathbf{e}_{j}\right)+\sum_{i, j=1}^{d} \nabla_{i} \nabla_{j} u_{0}(y) \phi_{j}\left(y+\mathbf{e}_{i}\right) \mathbf{e}_{i} .
\end{aligned}
$$

Combined with (3.32) the desired identity (3.31) follows.

Step 5d. Estimates of the vertical derivatives of $\boldsymbol{b}^{i j}$ and $z$.

We claim that

$$
\left\langle\left(\frac{\partial \boldsymbol{b}^{i j}(x)}{\partial y}\right)^{2}\right\rangle^{1 / 2} \lesssim(|y-x \bmod L|+1)^{-d}
$$

and

$$
\left\langle\left(\frac{\partial z(x)}{\partial y}\right)^{2}\right\rangle^{1 / 2} \lesssim I_{1}+I_{2}
$$

where

$$
\begin{aligned}
& I_{1}:=(|y-x \bmod L|+1)^{2-d-\gamma}\left(\left\langle|\nabla z(y)|^{2}\right\rangle^{1 / 2}+\mu_{d}(L)\left|\nabla^{2} u_{0}(y)\right|\right), \\
& I_{2}:=(|y-x \bmod L|+1)^{1-d}\left|\nabla u_{0}(x)-\nabla u_{0}(y)\right|,
\end{aligned}
$$

for some $\gamma>0$ depending only on $\lambda$ and $d$.

We start with (3.33). By the Cauchy-Schwarz inequality in probability, (3.30) turns into

$$
\begin{aligned}
\mid[\text { L.H.S. of }(3.33)] \mid \lesssim & \left\langle\delta\left(x-\mathbf{e}_{i}-y \bmod L\right)+\left|\nabla_{x_{i}} \nabla_{y} G_{L}\left(x-\mathbf{e}_{i}, y\right)\right|^{4}\right\rangle^{1 / 4} \\
& \times\left\langle\left|\left[\nabla \phi_{j}(y)+\mathbf{e}_{j}\right]_{y}\right|^{4}\right\rangle^{1 / 4} .
\end{aligned}
$$


The first term of the r.h.s. is estimated by the annealed estimate (3.9) of Lemma 3.3. For the second term we appeal to (3.28) with $q=4$, and to the bound (2.5) in Lemma 2.2 on the quartic moment of $\nabla \phi_{j}$ :

$$
\left\langle\left|\left[\nabla \phi_{j}(y)+\mathbf{e}_{j}\right]_{y}\right|^{4}\right\rangle \leq 2^{4}\left\langle\left|\nabla \phi_{j}(y)+\mathbf{e}_{j}\right|^{4}\right\rangle \lesssim\left\langle\left|\nabla \phi_{j}\right|^{4}\right\rangle+1 \lesssim 1 .
$$

The desired estimate (3.33) follows.

We then turn to (3.34). Based on (3.31), we first bound the l.h.s. in (3.34) by the sum of three terms:

$$
\begin{aligned}
\mid[\text { L.H.S. of }(3.34)] \mid \lesssim & \left\langle\left|\nabla_{y} G_{L}(x, y)\right|^{2}\left|F_{1}(y)\right|^{2}\right\rangle^{1 / 2} \\
& +\left\langle\left|\nabla_{y} G_{L}(x, y)\right|^{2}\left|F_{2}(y, x)\right|^{2}\right\rangle^{1 / 2}+\left\langle\left|\nabla_{y} G_{L}(x, y)\right|^{2}\left|F_{3}(y)\right|^{2}\right\rangle^{1 / 2} .
\end{aligned}
$$

We estimate the first and third terms on the r.h.s. by appealing to the quenched estimate of Lemma 3.2 and Jensen's inequality in probability:

$$
\begin{aligned}
& \left\langle\left|\nabla_{y} G_{L}(x, y)\right|^{2}\left|F_{1}(y)\right|^{2}\right\rangle^{1 / 2}+\left\langle\left|\nabla_{y} G_{L}(x, y)\right|^{2}\left|F_{3}(y)\right|^{2}\right\rangle^{1 / 2} \\
& \lesssim(|x-y \bmod L|+1)^{2-d-\gamma}\left(\left\langle|\nabla z(y)|^{2}\right\rangle^{1 / 2}+\max _{j=1, \ldots, d}\left\langle\phi_{j}^{2}\right\rangle^{1 / 2}\left|\nabla^{2} u_{0}(y)\right|\right) .
\end{aligned}
$$

Due to (2.4) in Lemma 2.2 the second moment of $\phi_{j}$ is bounded by $\mu_{d}(L)$, and thus we can control the r.h.s. by $I_{1}$. It remains to estimate the second term in (3.36). By appealing to the definition of $F_{2}$, the Cauchy-Schwarz inequality in probability, and (3.35) we have

$$
\left\langle\left|\nabla_{y} G_{L}(x, y)\right|^{2}\left|F_{2}(y, x)\right|^{2}\right\rangle^{1 / 2} \lesssim\left\langle\left|\nabla_{y} G_{L}(x, y)\right|^{4}\right\rangle^{1 / 4}\left|\nabla u_{0}(x)-\nabla u_{0}(y)\right| .
$$

In view of the annealed estimate (3.8) of Lemma 3.3 the r.h.s. is controlled by $I_{2}$ as desired.

Step 5e. Proof of (3.18) and thus (3.17).

By combining (3.34) and (3.33) we get

$$
\begin{aligned}
\text { [L.H.S. of }(3.18)] \lesssim & \left(\sum_{x \in \mathbb{T}_{L}}\left(\sum_{y \in \mathbb{T}_{L}}(|y-x \bmod L|+1)^{2(1-d)-\gamma}\left(\left\langle|\nabla z(y)|^{2}\right\rangle^{1 / 2}+\mu_{d}(L)\left|\nabla^{2} u_{0}(y)\right|\right)\right)^{2}\right)^{1 / 2} \\
& +\left(\sum_{x \in \mathbb{T}_{L}}\left(\sum_{y \in \mathbb{T}_{L}}(|y-x \bmod L|+1)^{1-2 d}\left|\nabla u_{0}(x)-\nabla u_{0}(y)\right|\right)^{2}\right)^{1 / 2} .
\end{aligned}
$$

Since $\gamma>0$, the discrete convolution kernel $x \mapsto(|x \bmod L|+1)^{2(1-d)-\gamma}$ has $\ell^{1}\left(\mathbb{T}_{L}\right)$-norm bounded independently of $L$ (even for $d=2)$, i.e. $\sum_{\mathbb{T}_{L}}(|x \bmod L|+1)^{2(1-d)-\gamma} \lesssim 1$. Hence, by the convolution estimate w.r.t. the $\ell^{2}\left(\mathbb{T}_{L}\right)$-norm, the first term on the r.h.s. is controlled by the r.h.s. of (3.18). It remains to treat the second sum and suffices to show that

$$
\left(\sum_{x \in \mathbb{T}_{L}}\left(\sum_{y \in \mathbb{T}_{L}}(|y-x \bmod L|+1)^{1-2 d}\left|\nabla u_{0}(x)-\nabla u_{0}(y)\right|\right)^{2}\right)^{1 / 2} \lesssim \mu_{d}(L)\left(\sum_{\mathbb{T}_{L}}\left|\nabla^{2} u_{0}\right|^{2}\right)^{1 / 2} .
$$

By the definition of the discrete gradient and periodicity we have for $i=1, \ldots, d$

$$
\left(\sum_{x \in \mathbb{T}_{L}}\left|\nabla u_{0}\left(x+\mathbf{e}_{i}\right)-\nabla u_{0}(x)\right|^{2}\right)^{1 / 2}=\left(\sum_{\mathbb{T}_{L}}\left|\nabla_{i} \nabla u_{0}\right|^{2}\right)^{1 / 2}
$$


which combined with the triangle inequality and periodicity yields

$$
\left(\sum_{x \in \mathbb{T}_{L}}\left|\nabla u_{0}(x+z)-\nabla u_{0}(x)\right|^{2}\right)^{1 / 2} \lesssim|z \bmod L|\left(\sum_{\mathbb{T}_{L}}\left|\nabla^{2} u_{0}\right|^{2}\right)^{1 / 2}
$$

for all $\mathbb{Z}^{d}$. We then use the triangle inequality in the form of

$$
\left(\sum_{x}\left(\sum_{z} X_{x z}\right)^{2}\right)^{1 / 2} \leq \sum_{z}\left(\sum_{x} X_{x z}^{2}\right)^{1 / 2}
$$

so that

$$
\begin{aligned}
& \text { [L.H.S. of }(3.37)] \\
& \qquad \quad\left(\sum_{x \in \mathbb{T}_{L}}\left(\sum_{z \in \mathbb{T}_{L}}(|z \bmod L|+1)^{2(1-d)} \frac{\left|\nabla u_{0}(x)-\nabla u_{0}(x+z)\right|}{(|z \bmod L|+1)}\right)^{2}\right)^{1 / 2} \\
& \stackrel{\substack{\text {-inequality } \\
\leq}}{\leq} \sum_{z \in \mathbb{T}_{L}}\left((|z \bmod L|+1)^{4(1-d)} \sum_{x \in \mathbb{T}_{L}} \frac{\left|\nabla u_{0}(x)-\nabla u_{0}(x+z)\right|^{2}}{(|z \bmod L|+1)^{2}}\right)^{1 / 2} \\
& \sum_{z \in \mathbb{T}_{L}}(|z \bmod L|+1)^{2(1-d)}\left(\sum_{\mathbb{T}_{L}}\left|\nabla^{2} u_{0}\right|^{2}\right)^{1 / 2} \cdot
\end{aligned}
$$

Evaluating the sum in $z$ on the r.h.s. yields the claimed estimate (3.37), recalling that $\mu_{d}(L)=\ln L$ for $d=2$ and $\mu_{d}(L)=1$ for $d>2$. This proves (3.18), and therefore the desired estimate (3.17) by Step 5a.

\subsection{Proof of Corollary 1.2}

Estimate (1.14) is a direct consequence of (1.13) and the estimate

$$
\max _{j=1, \ldots, d}\left\langle\left|\phi_{j}\right|^{2}\right\rangle \lesssim \begin{cases}\ln L & \text { for } d=2 \\ 1 & \text { for } d>2\end{cases}
$$

of Lemma 2.2.

\section{A. Proofs of the auxiliary lemmas}

\section{A.1. Proof of Lemma 2.1}

We adapt the arguments of [6], proof of Lemma 2, as for the proof of [10], Lemma 3 starting from [9], Lemma 2.3. We first introduce a couple of notations: Let $\left\{y_{n}\right\}_{n=1, \ldots, N}, N:=L^{d}$, be an enumeration of $\mathbb{T}_{L}$. For $n=1, \ldots, N$ define the average

$$
\langle\cdot\rangle_{\leq n}:=\prod_{1 \leq k \leq n} \int_{\Omega_{0}} \beta\left(d \boldsymbol{a}\left(y_{k}\right)\right) .
$$

Set $\zeta_{n}:=\langle\zeta\rangle_{\leq n}, \tilde{\zeta}_{n}:=\langle\tilde{\zeta}\rangle_{\leq n}$ for $n \geq 1$ and $\zeta_{0}:=\langle\zeta\rangle_{\leq 0}:=\zeta, \tilde{\zeta}_{0}:=\langle\tilde{\zeta}\rangle_{\leq 0}:=\tilde{\zeta}$. W.l.o.g. we assume that both $\zeta$ and $\tilde{\zeta}$ have zero expectation. We split the proof into two steps.

Step 1. Martingale decomposition. 
We claim that

$$
\langle\zeta \tilde{\zeta}\rangle=\sum_{n=1}^{N}\left\langle\left(\zeta_{n}-\zeta_{n-1}\right)\left(\tilde{\zeta}_{n}-\tilde{\zeta}_{n-1}\right)\right\rangle .
$$

Here comes the argument: Since $\left\{y_{1}, \ldots, y_{N}\right\}=\mathbb{T}_{L}$ we have $\langle\cdot\rangle=\langle\cdot\rangle_{\leq N}$ by $(1.10)$, and thus $\zeta_{N}=\langle\zeta\rangle=0$, $\tilde{\zeta}_{N}=\langle\tilde{\zeta}\rangle=0$ and $\zeta_{N} \tilde{\zeta}_{N}=0$. Moreover, by construction we have $\zeta_{0}=\zeta$ and $\tilde{\zeta}_{0}=\tilde{\zeta}$, and thus

$$
\zeta \tilde{\zeta}=\sum_{n=1}^{N} \zeta_{n-1} \tilde{\zeta}_{n-1}-\zeta_{n} \tilde{\zeta}_{n}
$$

The identity (A.1) then follows from taking the expectation of (A.2) provided we prove that

$$
\left\langle\left(\zeta_{n}-\zeta_{n-1}\right)\left(\tilde{\zeta}_{n}-\tilde{\zeta}_{n-1}\right)\right\rangle=\left\langle\zeta_{n-1} \tilde{\zeta}_{n-1}\right\rangle-\left\langle\zeta_{n} \tilde{\zeta}_{n}\right\rangle
$$

This last identity can be seen as follows: By definition we have

$$
\tilde{\zeta}_{n}=\langle\tilde{\zeta}\rangle_{\leq n}=\prod_{1 \leq k \leq n} \int_{\Omega_{0}} \tilde{\zeta} \beta\left(d \boldsymbol{a}\left(y_{k}\right)\right)=\int_{\Omega_{0}} \tilde{\zeta}_{n-1} \beta\left(d \boldsymbol{a}\left(y_{n}\right)\right) .
$$

Since $\zeta_{n}$ does not depend on $y_{1}, \ldots, y_{n}$, we have

$$
\zeta_{n} \tilde{\zeta}_{n}=\int_{\Omega_{0}} \zeta_{n} \tilde{\zeta}_{n-1} \beta\left(d \boldsymbol{a}\left(y_{n}\right)\right)
$$

Integrating both sides w.r.t. $\boldsymbol{a}\left(y_{k}\right), k \neq n$, yields $\left\langle\zeta_{n} \tilde{\zeta}_{n}\right\rangle=\left\langle\zeta_{n} \tilde{\zeta}_{n-1}\right\rangle$ and thus by symmetry $\left\langle\zeta_{n} \tilde{\zeta}_{n}\right\rangle=$ $\left\langle\zeta_{n-1} \tilde{\zeta}_{n}\right\rangle$, so that we obtain (A.3).

Step 2. Conclusion.

From [6], Step 2, Proof of Lemma 7, since $\langle\cdot\rangle$ is an $L$-periodic i.i.d. measure, for all $n \in\{1, \ldots, N\}$ we have

$$
\left\langle\left(\zeta_{n-1}-\zeta_{n}\right)^{2}\right\rangle \leq\left\langle\left(\frac{\partial \zeta}{\partial y_{n}}\right)^{2}\right\rangle, \quad\left\langle\left(\tilde{\zeta}_{n-1}-\tilde{\zeta}_{n}\right)^{2}\right\rangle \leq\left\langle\left(\frac{\partial \tilde{\zeta}}{\partial y_{n}}\right)^{2}\right\rangle
$$

Hence the claim follows from (A.1) and Cauchy-Schwarz' inequality.

\section{A.2. Proof of Lemma 3.2}

It suffices to consider the case $y=0$, since $G_{L}(x, y ; \boldsymbol{a})=G_{L}(x-y, 0 ; \boldsymbol{a}(\cdot-y))$ and due to the fact that the asserted estimate depends on $\boldsymbol{a}$ only through its ellipticity constant $\lambda$. We shall write $G_{L}(x):=G_{L}(x, 0 ; \boldsymbol{a})$ for brevity. We divide the proof into three steps. First we derive quenched estimates on the Green's function for $d>2$ from the quenched estimates of [6], Lemma 10 on the corresponding parabolic Green's functions. In the second step we prove the desired estimate for $d>2$ using the De Giorgi-Nash-Moser Hölder estimate, see [15], Theorem 5.2 in the discrete setting. We then deduce the estimate for $d=2$ from the estimate for $d=3$ using an argument by Avellaneda and Lin, see [2], proof of Theorem 13.

Step 1. Quenched estimate on $G_{L}$ for $d>2$.

Let $G_{L}^{\prime}(t, y)$ denote the periodic, parabolic Green's function considered in [6], Section 3.1; it is characterized as follows: For all $x \in \mathbb{Z}^{d}$ the function $G_{L}^{\prime}$ is the unique $C^{\infty}\left(\mathbb{R}, \ell^{\infty}\left(\mathbb{Z}^{d}\right)\right)$ solution of

$$
\left\{\begin{aligned}
\partial_{t} G_{L}^{\prime}(t, x)-\nabla \cdot \boldsymbol{a} \nabla G_{L}^{\prime}(t, x) & =0, \\
G_{L}^{\prime}(0, x) & =\delta(x \bmod L) .
\end{aligned}\right.
$$


Since both the initial data and the coefficient field are $L$-periodic, $G_{L}^{\prime}$ is in fact of class $C^{\infty}\left(\mathbb{R}, \ell^{1}\left(\mathbb{T}_{L}\right)\right)$. The elliptic Green's function $G_{L}$ can be formally obtained by integrating in time the mean free version of the parabolic Green's function:

$$
G_{L}(x)=\int_{0}^{\infty}\left(G_{L}^{\prime}(t, x)-L^{-d}\right) d t .
$$

To turn this into a rigorous argument one needs to prove that $t \mapsto G_{L}^{\prime}(t, x)-L^{-d} \in L^{1}(0,+\infty)$. This is a direct consequence of [6], Lemma 10: for all $\alpha>0$ we have

$$
\begin{aligned}
0 \leq G_{L}^{\prime}(t, x) & \lesssim(t+1)^{-\frac{d}{2}}\left(\frac{(|x \bmod L|+1)^{2}}{t+1}+1\right)^{-\frac{\alpha}{2}} \text { for } t \lesssim L^{2}, \\
\left|G_{L}^{\prime}(t, x)-L^{-d}\right| & \lesssim L^{-d} \exp \left(-c_{0} \frac{t}{L^{2}}\right) \quad \text { for } t \gtrsim L^{2} .
\end{aligned}
$$

For $d>2$, this directly yields

$$
\left|G_{L}^{\prime}(x)\right| \leq \int_{0}^{\infty}\left|G_{L}(t, x)-L^{-d}\right| \mathrm{d} t \lesssim(1+|x \bmod L|)^{2-d} .
$$

Step 2. Quenched estimate on $\nabla G_{L}$ for $d>2$.

We now deduce (3.7) from (A.5) for $d>2$. Recall that it suffices to prove the estimate for $y=0$ and that we write $G_{L}(x)=G_{L}(x, 0)$ for brevity. Fix a radius $2 \leq R \sim 1 \ll L$. For $|x \bmod L| \leq 2 R$ the r.h.s. of $(3.7)$ is of order 1 so that the estimate directly follows from the combination of (A.5) with the discrete estimate $\left|\nabla_{i} G_{L}(x)\right| \leq\left|G_{L}\left(x+e_{i}\right)\right|+\left|G_{L}(x)\right|$. Hence, it suffices to consider the case $|x \bmod L| \geq 2 R$. For all $y^{\prime} \in \mathbb{T}_{L}$ and $r>0$, set $B_{r}\left(y^{\prime}\right)=\left\{y^{\prime \prime} \in \mathbb{T}_{L},\left|y^{\prime}-y^{\prime \prime} \bmod L\right| \leq r\right\}$. Using the elementary inequality

$$
\left|\nabla G_{L}(x)\right| \leq \sqrt{d} \underset{B_{1}(x)}{\operatorname{osc}} G_{L},
$$

this turns into an estimate of the oscillation of $G_{L}$ on $B_{1}(x)$. Noting that $G_{L}$ satisfies

$$
\nabla^{*} \boldsymbol{a} \nabla G_{L}=L^{-d}
$$

in the set $B_{\mid x} \bmod L \mid / 2(x)$ (which does not contain $0 \bmod L$ ), one may appeal to the De Giorgi-Nash-Moser Hölder estimate. In particular, by [15], Theorem 5.2, there exists $0<\gamma<1$ depending only on $\lambda$ and $d$ such that

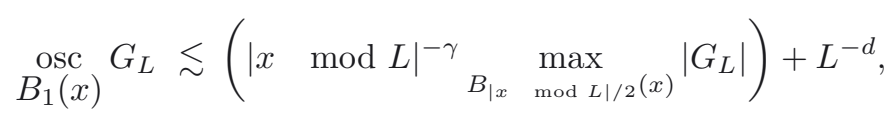

Combined with (A.5), this yields (3.7) for $d>2$.

Step 3. Quenched estimate on $\nabla G_{L}$ for $d=2$.

Following [2], proof of Theorem 13, we derive the estimates for $d=2$ from the estimate for $d=3$. To distinguish quantities in different dimensions we use the superscripts ${ }^{(2)}$ and ${ }^{(3)} ;$ e.g. $\mathbb{T}_{L}^{(2)}$ denotes the 2-dimensional torus. To a given two-dimensional, $L$-periodic coefficient field $\boldsymbol{a}^{(2)} \in \Omega_{L}^{(2)}$ we associate a three-dimensional coefficient-field $\boldsymbol{a}^{(3)} \in \Omega_{L}^{(3)}$ via

$$
\boldsymbol{a}^{(3)}\left(x, x_{3}\right):=\operatorname{diag}\left[\left[\boldsymbol{a}^{(2)}(x)\right]_{1},\left[\boldsymbol{a}^{(2)}(x)\right]_{2}, 1\right] \quad\left(x, x_{3}\right) \in \mathbb{Z}^{2} \times \mathbb{Z}=\mathbb{Z}^{3} .
$$

In the following we use the shorthand notation $G_{L}^{(2)}(x):=G_{L}^{(2)}\left(x, 0 ; \boldsymbol{a}^{(2)}\right)$ and $G_{L}^{(3)}\left(x, x_{3}\right):=$ $G_{L}^{(3)}\left(\left(x, x_{3}\right),(0,0) ; \boldsymbol{a}^{(3)}\right)$. It is elementary to check that

$$
G_{L}^{(2)}(x)=\sum_{x_{3} \in([0, L) \cap \mathbb{Z})} G_{L}^{(3)}\left(x, x_{3}\right)
$$


and thus

$$
\nabla_{i} G_{L}^{(2)}(x)=\sum_{x_{3} \in([0, L) \cap \mathbb{Z})} \nabla_{i} G_{L}^{(3)}\left(x, x_{3}\right) \quad \text { for } i=1,2 .
$$

The quenched estimate (3.7) for $d=3$ then turns into

$$
\begin{aligned}
\left|\nabla_{i} G_{L}^{(2)}(x)\right| & \leq \sum_{x_{3} \in([0, L) \cap \mathbb{Z})}\left|\nabla_{i} G_{L}^{(3)}\left(x, x_{3}\right)\right| \\
& \lesssim \sum_{x_{3} \in([0, L) \cap \mathbb{Z})}\left(1+\left|\left(x, x_{3}\right) \quad \bmod L\right|\right)^{-1-\gamma} \\
& \lesssim \sum_{x_{3} \in([0, \infty) \cap \mathbb{Z})}\left(1+|x \bmod L|+x_{3}\right)^{-1-\gamma} \\
& \lesssim(1+|x \bmod L|)^{-\gamma} \lesssim(1+|x \bmod L|)^{-\gamma}
\end{aligned}
$$

which is nothing but (3.7) for $d=2$.

\section{REFERENCES}

[1] G. Allaire and M. Amar, Boundary layer tails in periodic homogenization. ESAIM: COCV 4 (1999) 209-243.

[2] M. Avellaneda and F.-H. Lin, Compactness methods in the theory of homogenization. Commun. Pure Appl. Math. 40 (1987) 803-847.

[3] A. Bourgeat and A. Piatnitski, Estimates in probability of the residual between the random and the homogenized solutions of one-dimensional second-order operator. Asymptotic Anal. 21 (1999) 303-315.

[4] J.G. Conlon and T. Spencer, Strong convergence to the homogenized limit of elliptic equations with random coefficients. Trans. $A M S$, in press.

[5] A. Gloria, Fluctuation of solutions to linear elliptic equations with noisy diffusion coefficients. Commun. Partial Differ. Eq. 38 (2013) 304-338.

[6] A. Gloria, S. Neukamm, and F. Otto, Quantification of ergodicity in stochastic homogenization: optimal bounds via spectral gap on Glauber dynamics. MPI Preprint 91 (2013).

[7] A. Gloria, S. Neukamm and F. Otto, Approximation of effective coefficients by periodization in stochastic homogenization. In preparation.

[8] A. Gloria and F. Otto, Quantitative results on the corrector equation in stochastic homogenization of linear elliptic equations. In preparation.

[9] A. Gloria and F. Otto, An optimal variance estimate in stochastic homogenization of discrete elliptic equations. Ann. Probab. 39 (2011) 779-856.

[10] A. Gloria and F. Otto, An optimal error estimate in stochastic homogenization of discrete elliptic equations. Ann. Appl. Probab. 22 (2012) 1-28.

[11] R.J. Leveque, Finite difference methods for ordinary and partial differential equations. Society for Industrial and Applied Mathematics (SIAM), Philadelphia, PA (2007).

[12] S.M. Kozlov, The averaging of random operators. Mat. Sb. (N.S.) 109 (1979) 188-202, 327.

[13] R. Künnemann, The diffusion limit for reversible jump processes on $\mathbb{Z}^{d}$ with ergodic random bond conductivities. Commun. Math. Phys. 90 (1983) 27-68.

[14] D. Marahrens and F. Otto, Annealed estimates on the Green's function. MPI Preprint 69 (2012).

[15] S.J.N. Mosconi, Discrete regularity for elliptic equations on graphs. CVGMT. Available at http://cvgmt.sns.it/papers/53 (2001).

[16] A. Naddaf and T. Spencer, Estimates on the variance of some homogenization problems. Preprint (1998).

[17] H. Owhadi, Approximation of the effective conductivity of ergodic media by periodization. Probab. Theory Relat. Fields 125 (2003) 225-258.

[18] G.C. Papanicolaou and S.R.S. Varadhan, Boundary value problems with rapidly oscillating random coefficients. In Random fields, Vol. I, II (Esztergom, 1979), vol. 27 of Colloq. Math. Soc. János Bolyai. North-Holland, Amsterdam (1981) 835-873.

[19] V.V. Yurinskiǔ, Averaging of symmetric diffusion in random medium. Sibirskii Matematicheskii Zhurnal 27 (1986) 167-180. 